\title{
MATHEMATICS, QUANTIFIERS, CONNECTIVES, MULTIPLE MODELS ${ }^{1}$
}

\author{
Rosanna Festa \\ Alumni NoiSapienza \\ Università La Sapienza \\ Reggio Calabria, Italia
}

DOI: $10.37648 /$ ijrst.v10i02.005

Received: 02 ${ }^{\text {nd }}$ March, 2020; Accepted: 28 $8^{\text {th }}$ March, 2020; Published: $30^{\text {th }}$ April, 2020

\begin{abstract}
This focus evolves around the concept of mathematics and its components and the importance of connectives for mathematics applied to calculators. Symbols and synthesis are enrolled to notice their rule in mathematics and general intelligent machines.

Keywords - Language; Models; Data; Galileo; Mathematics.
\end{abstract}

\section{FIRST CHAPTER : DETERMINATION OF THE MAIN FORMS OF LANGUAGE}

In the logarithm's function, on demonstrate that it exists a problem, for definition, of elaboration and direct calculation ${ }^{1}$. For the semantics of the logical systems, we define some circular functions, that are the direct calculation of fundamental limits in the most high value. The system of two equations, then, provides different phases of numeric calculation: whether taking it to the denominator, though the main proceeding of the calculator, or to the denominator, extracting of it the root (in mathematical logics it is affirmed a system of elaboration can exist according to whom we applicate the Lowenheim-Skolem's theorem otherwise the Church's theorem, that is therefore a programming of the weak completeness theorem. ${ }^{2}$. In theory, for elaborative models of second and third order in mathematics we take reference to the theorems of the calculation of a limit, the circular functions, the exponential functions, and to the vector's function. In mathematical logics, starting from an axiom of calculation in flat geometry, that involves the Cartesian coordinates, it is possible to establish that, in difference with the flats, the function presents an exponential degree when on traits of a function composed at the limit of the function given by the limit's theorem. This is the main problem of the multiple models in mathematics and the quantifiers in mathematical logics.

\footnotetext{
${ }^{1}$ How to cite the article: Festa R., Mathematics, Quantifiers, Connectives, Multiple Models, IJRST, Apr-Jun 2020, Vol 10, Issue 2, 32-65, DOI: http://doi.org/10.37648/ijrst.v10i02.005
} 
It is possible to speak about, in the system pure theory, of mathematics of logics, but it is justified to assert also the applied logics to mathematics, but in the logics the displaced variables are discussed, the logics is mathematics of the physical quantities if we leave out the mathematical and the logic variable. For this fact, the division between mathematics and logics is very important to deduct many physical cases and many chemical cases, but even to abstract some important consequences in phenomenology, for example, as the system that allows a continue function and if they have been always analyzing according to systemic formalities in mathematics (of course of notquantitative nature). Whilst the logics development takes of importance to the shapes of argumentations, the attitude of the modern mathematical logics would be making a précis of the phrase combinatory study of the content, and obviously, the first has retained the most important owing to the parithetic synthesis.

In the words of programming of logics, on refers that it is necessary to achieve to some fundamental results, the first for the concerning the brief analysis, the second on the other hand for the concerning the formal system. Then: 1) the putative demonstrations of the universal acceptance of the formulas in logics of first order can be submitted to the algorithmic verify of their strength. With a technical expression it is sustained that the demonstrations language is primitive and ricorsive. Essentially, that is equally compared to the Godel's completeness theorem; it nevertheless is formulated generally speaking in a way to make clear it has nothing to do with the algorithms. 2) The language of the accepted formulas in the logics of first order is not deciding, but half-deciding, this has a consequence it exist an algorithm that is able to evaluate the formula's acceptance. In the case in that the formula will be validated the algorithm is in a position to terminate his shape in analytic giving back as a proof the demonstration of his validity, on the contrary, if the formula is not validated, the algorithm is not in a position to notice and it continue to put calculations (it is said that it diverges), without to supply into an execution never a reply. For this the language of formulas is ricorsively enumerable, without to leave out any element of the logics of second order. 3) If we distinguish the enumerable language is avoided by reason of the programming of logics of the first terms. The principle of relationship is a mathematical rule for the pure sciences and the applied sciences. Obviously, it is a matter of a quantifier and a measure relatively to the inductive-statistical problem, to the combination, and to the diagrams. That is a question of relationships studied from the mathematician through links of inference that complete the description, for example, in Frege and Russell, they are not quantitative, but they are qualitative, still it is necessary to leave out of them the variables by simplifying. It is given, for example, the reasoning about the formal relationships ${ }^{3}$. Analyzing the various criterions or the terminology, we will have above all the position for that even in Cantor the study of mathematics is first of all a study of relationships that can be of different nature. "First of all we can considerate reals the whole numbers in the measure in, according to some definitions, they hold in our intellect a place absolutely determined, they are exactly separated from all the other constituting parts of our thought, stay with them in determined relationships and modify then the substance of our spirit in definitive way; it would be accorded to me to call intra-subjective or immanent this kind of reality of our numbers. But it is possible even to admit a reality to the numbers in the measure in that they are to be considered expression or image of processes and relationships of the external world that remains in front of the intellect.[..] I call trans-objective or transient this second kind of reality of the whole numbers (Cantor, 1992:97). For Cantor, "the sets theory belongs to the pure mathematics and not to that applied because according to him every application requires some metaphysical inquires and, seen that the sets theory has not to control the "transient truth" of own assertions, that is it have not to lean to any metaphysics, it is free, or "pure"; for Peirce, instead, the application begins with the logic insertion, that is when a comparison is formulated between two greatness implicating a conceptualization or a generalization of the pure "mathematic gesture": the application begins from the definition of whole and from the fact it would have a certain greatness ${ }^{4}$.

We have then syntagmatic periods of first and second type, obtaining through an analysis process of the hidden variables. A system, in fact, can be of computational type or mechanical or dielectric type. Boltzmann, by the way, lays down two or three possible alternatives: the division in the Hertzian mechanics $^{5}$, or the chemical nomenclature ${ }^{6}$. The chemical composites (with density or approximation variables) set up millesimals in the altimetric scale, whether for the motion principle, or the subdetermination of complex atoms describing in terms of super-structured compounds, of which has spoken even Herbart for the classification between particles physics 
and the macro-molecular chemistry toward the QCD, that describes the quantic chromo-dynamics, or the quantum chromo-dynamics, so as some substances in optics, in the first case of analysis we have a mathematical and computational physics, in the second case a model of chemical analysis. In the third case, the computer is reduced to a scale of algebraic functions and altimetric-notational ${ }^{7}$ that accomplish an ordinary process, the calculator makes known then the computer in analytic way, according to the inductive methodology or according to that hypothetic-deductive. For the refraction, Descartes is inspired even by Cassirer $^{8}$, but it elaborate above all considerations on the elaborating of series of Aristotle with extended properties, based on syntagmatic properties, that were noticed in the Manierism for the Dioptrics of the notable calculators. In fact, the method utilized in chemistry, reduces the scale of a fraction to the maximum degree of measurement for the macromolecular chemistry and considering them chemical and deflative properties, in that matter properties. For that, the method of algebraic analysis, of the Baconian manner, provides a type of data very fast. The second method, of programming in logics, provides a transitive method, of a graduated type, otherwise in hypothetic-deductive and analytical type, whilst the research method in series provides two solutions: the logic of the standard elaboration, and the elaboration of casual models ${ }^{9}$, of complex systems on the model of Heyting's algebra, the rules of inference of the principle of bivalence, the logic programming. In arithmetic analysis we utilize numeration series and numeration of arithmetic function series, according to the type of partition, carry out a distribution in parts or half-wholes until the inducted period in the partition coefficients, if it exist, calculating from one side the whole coefficient and from the other side the report of the semi-axis. In a form of numeration, we can to obtain a whole in numeration series in a "preestablished" way, either that it would be of an orthogonal matrix or that it would be of an orthospheric matrix. In fact, if the numerals series calculates, obviously, numbers of fractions, it is necessary to classify in order the matrix series and the records sequences. For this work, they exist mono-variant classes in trigonometry and the geometry of indefinite integration. Then, in logarithmic curve, we calculate the outcome's phase level of oscillations of a point of departure, that can be a calculation in the data or a variation. For examples, in quadrangular measurement, the physical object must to foresee a statistic error that have to be corrected the most possible, as for example in the photoplanimetry, obtaining data in series, otherwise data more remarkable for quadrangular objectives, that take part of the distortion axis, noticed that can be also a semi-axis ${ }^{10}$.

We take the Weierstrass's construction, that is constituted on a perpendicular straight line to the axis and it can be the resulting of a relationship of two noticed semi-axis, it can exist then an any point in way to connect the straight line with the obtained measurement through the spheric diopter. In optics, in fact, existing the refraction in a point, it is possible not to obtain a whatever data, but a method of calculation that must be applied to the object in a phase of geometrical construction, if that object do not have determined characteristics. In the composition technics, for example in the cyclic accelerator "drift-tubes", in binary phases, to the basis of some types of variations, we distinguish the stator of electrodes collectors, the reaction units of the electrodes of charge. This happens because it however exist a geometric part, but the direction can vary on behalf of the distance. To follow the inverse process, we utilize different types of angulation, above all in the partition's theory, often in the last Fermat's theorem of the equivalent system, in the Cartesian hypothesis, and in the quantic mechanics (QM), according to decimal partitions or to standard numbers, for example, from 20-24. In molecular physics, they however exist, even in this case, some constraints, because the variable is measured in accordance with the acceleration period, having a data flow, or a system fraction in the Bremsstrahlung. The problem remains to determine what information leaves from a point of exchange data, on the straight line of exchange, and every atom contains ones minimum angulations and previous values to the m.c.m. In fact, A sends the information along the vertical column defined from its values $\mathrm{x}$ and $\mathrm{y}$, until the same maximum distance. In the Galileo's studies of Koiré, editor of the Newtonian studies, are compared the Descartes physics and that of Galileo, here the author sustains the fact that Descartes had been able to formulate the inertia's principle through stationarity's principles, or rather making a comparison through a logic principle of inference through means of transmission of a body, as for example in optics, and studying it through geometrical properties, this problem, was been confronted also from K. Popper, in the Logics of the scientific discovery, on behalf of the relevance of the physical system object of the analysis. Descartes speaks about nature how "it must" be, unlike Galileo, that continues to ask himself as "it is" 
In practice, Descartes formulates the hypothesis of a calculator widely accepted for the theoric and practical applications, utilizing, for example, the conic section. In cause of the problem, Popper from a decision system of the calculation of the speeds, or rather through an hypothesis of geometrisation, that is mostly plausible, we applicate the logics method through some subsections, that are nothing but the explicandum of the mathematical rules ${ }^{12}$. It exist then the problem how to determine for the explicans the frequency of a calculator $^{13}$ determining in them the calculability through rational methods, then can determine the frequency, the place or rather the given instant in that is registered a sequential system ${ }^{14}$. It is possible to determine, in fact, how the frequency is assumed as basis for the statistic series, equiprobable, but it is not possible to determinate the result of a selection, of an independence or an irrelevance of two frequencies, it remains the problem how to put on, if however it is a matter of stable numerations ${ }^{15}$, as would be the relationship between statistic series that varies from the numeration system to the denomination in equidistant relationship or rather through elevations to $1 / 14$, that allows to the information to "isolate" the mechanic data from a physical or geometrical property according to an adaptable method. These evaluation's information are important for the complex systems whether for the "corroboration's degree" provided by Popper, or the adaptable function of the calculator. They exist, in fact, methods at finite sequence for the informatics data and for elaboration data ${ }^{16}$, adopted through the moltiplication's theorem for the methods of identification of the pair numbers ${ }^{17}$. Leaving from physical and theoretical presupposes regarding the philosophical aspects of the physical theories, in philosophy of physique, that defines the entity of matter, energy, space and time, we leave from the space's notion in physics, and then the philosophical questions regarding the space comprehended the problem of absolute space or the space purely relational, and if the space has an inherent geometry, otherwise the space's geometry is only a convention. Then, they exist three physical systems to effect a space's formalization in philosophy and in physique. From one side the Euclidean geometry, from the other the not-Euclidean geometry that excludes some Euclidean geometries or the physical systems. Beginning from the first analysis, the Cartesian system settled in infinitary mathematics is a system of coordinates, called Cartesians, that go away from the straight line. From a substratum of mutations, afterword in philosophy of the physique we can have two relational entities, that in statistic mechanics we call variables, that are at the basis of the determinism. From one side, in statistic mechanics, we have a decoherent system (logics), from the other the analysis of a complex phenomenon, languages, and relationships. For example, we utilize for the analysis an enumeration method, or methods that are descriptive and, thus, come near the geometry. For the comparison systems tied to these complex phenomenon, it is necessary an order definition. Gauss sustained that from a substratum of the geometry, and then of the analytic mechanics, it is possible to go through physical criterions. Einstein asserted, instead, that it is necessary to understand what we assign to the relativity through physical systems and what to the physical systems through mechanical systems. In mechanics, in fact, wave and particle take part of physical systems ${ }^{18}$. They are obtained however a physical system and a dual system though to achieve a measurement. The problem is given in the fact that the systems in applied physics provide different types of classification and distinction, for example, beginning from the MaxwellBoltzmann's functions, from the Bose-Einstein's function to the physical law of Fermi-Dirac. This last indicates that exist sectors of the applied statistics, as the computational physics, in that on calculate the mathematical relationships of the whole.

K. Popper, Logics of the scientific discovery, The character auto-corrective of the science, p. 225. Logics of the scientific discovery, p. 156. "A collective is, using an inexact language, a sequence of events or occurrences that is passing, in line of principle, to be continued indefinitely: for example a sequence of throwings done with a die that it is supposed to be indestructible. Each of these events has a certain character or property: for example, the die can show the face of the five, and to have so the property five. If we take all the throwings having the property five that are appeared until a certain number of the sequence and we divide their number for the total number of the throwings until that element (that is to say the ordinal number of the element in the sequence) we obtain the relative frequence of the five until that element. If we determine the relative frequency of the five for each element of the frequency, we obtain, in this way, a new sequence: the frequency of the relative frequencies of five. The frequency of the frequencies is distinct from the original sequence of events who corresponds, and that can be called in the sequence of the events" or "the frequency of the properties". By giving a simple example of connective I have chosen that we can call 
an "alternative". [..] Now, the convergence's axiom (or "limit's axiom") postulates that, by degrees that the sequences of events became more long, the sequence of the frequencies holds out to a definite limit".

p. 120-126. For what is regarding the universality's limits and the precision's degrees and in general the logic fields in the measurement of standard physical constants.

p. 46. "We can distinguish among two types of universal synthetic assertions: the assertions "strictly universals" and the assertions "numerically universals". Until here, speaking of universal assertions - of theories or nature's laws - I had in mind the strictly universal assertions. The assertions belonging to the other type, that is these numerically universals, are, in reality, equivalent to some irregular assertions or conjunctions of singular assertions and they will be here classified between the regular assertions. It would be possible to compare, for example, the two following assertions: a) for all the harmonic oscillators is time that their energy do not fall never under a certain quantity (that is: $h v / 2$ ); and b) for all the human beings at present living on the Earth is true that their height do not surpass never a certain measure (we suppose 2,40 $\mathrm{m})$. The formal logics (included the symbolic logics) do not occupy just of the deduction's theory, it traits these two assertions in the same way, as universal assertions (implications "formals" or "generals"). I think instead that would be necessary to accentuate the difference among them. The assertion a) claims to be true in every place and in every time. The assertion $b$ ) refers itself only to a finite class of specific elements, limited to a spatial-temporal individual area (or particular area). The assertions of this last type can, in line of principle, be replaced from a conjunction of singular assertions; in fact, given a time enough long, can be enumerated all the elements of the finite class taken in consideration. It is the reason for which in cases like this, we speak about "numeric universality". On the contrary, the assertion a) on the oscillators can be not replaced from the conjunction of a finite number of regular assertions in a definite special and temporal area; or, rather it could be replaced from this conjunction only if it would be assumed that the world is limited in the time and in the space and that in this exists just a finite number of oscillators. But we do not do any assertion of this kind, especially, we do not do it when we definite the concepts of physics. Rather, we take in consideration an assumption of the kind of a) as an all-assertion, that is as an universal assertion around an unlimited number of individuals. It is obvious that interpreted in this manner, it can be not replaced from the conjunction of a finite number of regular assertions".

p. 13. Between many objections that will be probably raise against the point of view proposed in this chapter, the most serious is perhaps the following. Anyone could say that, refusing the inductive method, deprived the empirical science of what seems the characteristics most important; and this means that I eliminate the barriers that separates the science from the metaphysic speculation. To this objection on replays that the principal reason for that I refuse the inductive logics is precisely this: that it do not provides an appropriate countermark to distinguish the empirical character, not metaphysic, of a system of theories; or, in other words, that not provides an appropriate "criterion of demarcation". I call problem of demarcation the problem to find a criterion that would enable us to distinguish between the empirical sciences from one side and the mathematics and the logics, and so also the "metaphysical" system for the other. This problem was known to Hume that tried to resolve it. With Kant it became the central problem of the knowledge's theory. If, following Kant, we call "Hume's problem" the induction's problem, we have been calling "Kant's problem" the demarcation's problem. Afterword: "In the literature of the physics can be found some cases, referred by serious researchers, of the realizing of events that could be not reproduced because subsequent controls had lied to negative results. Wellknown example of a case of this kind, happened in recent times, is the positive result, of the Michelson's experiment discovered by Miller [...] (p. 28).

Through the multiplier's theorem, we are present to a models study to determine not predictable numerations, from the "criterions of demarcation" it passes through, in fact, to the study of new variables and to the project of a new theory of probability. "The two axioms or postulates, formulated by von Mises with the purpose to define the concept of collective, are been subdued to violent critics; and I believe that such critics will be not quite justified. In particular, against the combination of the convergence's axiom and the disorder's axiom are been raise objections based on the impossibility to admit the application of the mathematical concept of limit or convergence to a sequence that for definition (that is, as the disorder's axiom) must be not subjected to any law or mathematical rule. In fact, the mathematical limit is only that a characteristic property 
of the mathematical rule or law that defines the sequence. It is simply a property of this rule or law: if, for whatever fraction choose near as it wants to zero, it exists in the frequency a such element that all the elements subsequent to it differs of a minor quantity of that fraction from some defined value, that is called their limit. To confront these objections it is proposed to avoid the combination of the convergence's axiom with that of disorder, and to refine just the convergence, that is the existence of a limit [..] (p. 159).

The terminology of Leibniz constitutes some models of mathematical connection and they constitutes a central knot for the theorem of half-decision in logics. "A particular kind of formal object forms physical objects, which we take to include both physical events and physical systems - the latter is a term generic in (philosophy of) physics. Physical systems in turn comprise material objects, but not conversely; physical systems are more general than material objects in that they can be built out of material objects, fields, radiation, space and time; the latter four items are tipically "not material", yet indisputably "physical". An elementary particle can be characterized simply as a physical system having no proper subsystems, such as the leptons, quarks, and gauge bosons. Within quantum theory, one can also characterize kinds of elementary particles mathematically, in Wignerian fashion: that is, in terms of irreducible representations of the spacetime symmetry group, the Galilei group in the case of $\mathrm{QM}$, and the Poincaré group in the case of relativistic quantum field theory. When the material object is not an elementary particle and has a non-vanishing size, one speaks of a material body. We call physical objects in a set absolutely discernible if of every object there is some physical property that it has but all the others in the set lack, and relationally discernible if for every object there is some physical relation that discerns it from all others (cf. section 4 for rigorous definitions) . An object is indiscernible if it is both absolutely and relationally indiscernible, and hence discernible if it is discernible either way of both ways. The terms "qualitative", "quantitative", and "numerical discernibility" also abound in the literature: they can be defined in terms of our notions: physical objects are qualitatively discernible if they are discernible; they are quantitatively discernible, or synonymously numerically discernible; if they are not identical. Often, we call objects that are absolutely discernible from all other objects individuals; those that are only relationally discernible from all other objects we call relational. Frequently, one encounters talk of a physical object "having an identity", which we shall accommodate as the property that discerns the object absolutely (if it is absolutely discernible); than relational do not "have an identity" but individuals do. To individuate an object means to discern it absolutely; then individuals can be individuated, relational cannot. Finally, particles are distinguishable if they can be individuated. Next, we give three versions of Leibniz's principles for physical objects. The principle of Identity of absolute undiscernible (PII-A) state that no two physical objects are absolutely undiscernible. The principle of identity of relational undiscernible (PII-R) state that no two physical objects are absolutely and relationally indiscernible; or synonymously, two physical objects are numerically discernible only if they are qualitatively discernible. All converse statements are uncontroversial tautologies: no physical object can be discerned from itself - the indiscernibility of identical, also known as Leibniz's law. The relevant logical relations between PII, PII-A and PII-R are as follows. Obviously, PII-A and PII-R each are sufficient for PII,

$\mathrm{PII}-\mathrm{A} \rightarrow \mathrm{PII}$ and PII-R $\rightarrow$ PII,

which makes even their disjunction sufficient for PII. So if PII fails, than both PII-A and PII-R fail. Since absolute discernible are always relational discernible see equation (40) and the sentence proceeding it - one quickly proves that PII-R is also necessary for PII, which implies with Equation (1) that PII and PII-R stand or fall together,

PII $\leftrightarrow$ PII-R.

But PII-A is not necessary for PII, i.e. $\neg(\mathrm{PII} \rightarrow$ PII-A), which implies that it is a genuine logical possibility that PII-A fall whilst PII stands tall,

PII $>\neg$ PII-A.

The main conclusion of the current paper will be that similar elementary particles turn this logical possibility into a physical actuality: they are non-identical absolute indiscernibles. In the Discourse on metaphysics, Leibniz was the first to discuss PII-A elaborately and to apply it to "substances"; in several places, Leibniz defends (as we would put it today) a reduction of relations to properties, which makes mentioning relations in discernibility otiose (see (Russell [1937], pp. 13-5) and (Ishiguro [1990], pp. 118-22, 130-42) for Leibniz's struggle with relations). 
When not all relations reduce to properties, and we thus have to consider properties and relations separately and independently, then as a matter of logic, PII-R is as much in play as PII-A, and PII as stated is mandatory. Massimi ([2001]), holds that a version of Leibniz's principle which considers only relations irreducible to properties (a strengthened version of our PII-R) as the one that is applicable in QM. Let us end by noting that logically speaking one could refine "relational discernibility" to "n-discernibility", meaning that the objects are discerned by some n-ary relations. Then an infinite hierarchy of indiscernibility principles ensues, each one logically weaker that the next one. Since we shall not need this, we leave it."

The symmetry's operators take part of the measurers of symmetry in the composite physical systems, being constituted by physical operators that isolate the component of the symmetry's group, besides to represent models of informatics and compared grammar, it constitutes an only model for the parts of the physical systems, and then the physical objects ${ }^{19}$. In the multiple models, they exist then linear operators, and some distributions in mathematics of the theory according to a constant ${ }^{20}$.

The question is if effectively exists a method of separation of the functions of variables ${ }^{21}$ though the position of a flat geometry, and then of the curves lines where we develop an equation from which it would results the conjunction of such lines. As it has been affirmed for the formal logics, a system $\mathrm{E}^{\circ} / \mathrm{c}^{2}$ can exist not, because it traits not of a projection of curves, otherwise Einstein would refer to a linear association, in QEM, and it traits of a method very much utilized in the molecular physics ${ }^{22}$. Generally speaking, $\mathrm{E}^{\circ} / \mathrm{c}^{21}$ developed in mathematical analysis is the result of deriving or a difference of function ${ }^{23}$. "The definition says to us that we can define than a round by means of $\varepsilon$, that is the round from a- $\varepsilon$ to $a+\varepsilon$ such that, for all the arguments in this round, the value of the function ties inside the round that has for extremes $f(a)$ and $-\sigma$ and $\mathrm{f}$ (a) and $+\sigma$. If this happens, whatever would be $\sigma$, the function is continue for the argument a. Until here we have not defined the limit of a function for a given argument. If we would have done it, we would have defined the continuity of a function in a different way: a function, in fact, is continue in a point if its value is the same of the limit of its values when the variable of the function approximates to the argument from right or left. [..] The general rule is that the functions oscillates and that, given a round of a number, how much small this round would be, on have a certain whole of values of the function for the arguments contained in this round. Given that this is the general rule, we examine it. We consider that happens when the variable of a function holds out to an argument a from the inferior values. We consider, that is, that happens for arguments contained in the interval comprised from a- $\varepsilon$ to a, where $\varepsilon$ is a number that, in reality, will be very small. The values of the function for arguments from a- $\varepsilon$ to a (excluded) will be a whole of real numbers that will define a certain section of the whole of the real number, the section that is to say formed by the numbers that are not greater of all the values of the function for the arguments from a- $\varepsilon$ to a"24.

The answer obviously is affirmative, because it take in consideration the argument, given that the function presents inferior values. "The classes theory is less complete of the description's theory, and they exist good reasons to consider the definition of classes that we will give as not completely satisfying [..]. Every class, as it is explained in the chapter $\mathrm{II}^{25}$, defines with a certain propositional function that is true for the members of the class and it is false for the other thinks. But if a class would be defined with a certain propositional function, then it would be possible equally to define with every other propositional function that would result true when the first is true and false when the first is false. For this reason a class can be not identified with a propositional function rather than another. Given in fact a propositional function, they exist always many other functions that are true when it is true and false when it is false. When it happens, we say that the two propositional functions are "formally equivalents". Two "propositions" are "equivalents" when are both true or false; two propositional functions $\mathrm{f}(\mathrm{x}), \mathrm{p}(\mathrm{x})$ are "formally equivalents" when $\mathrm{f}(\mathrm{x})$ is always equivalent to $\mathrm{p}(\mathrm{x})$. it is properly the fact that they exist other functions formally equivalent to a given function that gives back impossible the identification of a class with a function; in fact, we want that the classes are such that it would be excluded the possibility of two different classes that would have exactly the same members then, two functions formally equivalent will to determine the same class ${ }^{26}$.

The theory proposed by Ghirardi, Rimini and Weber ([1986]) is in agreement with the predictions of nonrelativistic quantum mechanics as far as all present experiments are concerned (Bassi and Ghirardi [2003]); 
for a discussion of future experiments that may distinguish this theory from quantum mechanics, see Section V of Bassi and Ghirardi ([2003]). according to the way in which this theory is usually presented, the evolution of the wave function follows, instead of Schrodinger's equation, a stochastic jump process in Hilbert space. We shall succinctly summarize this process as follows. Consider a quantum system described (in the standard language) by an $\mathrm{N}$ "particle"1 wave function $\psi=\psi(\mathrm{q} 1 . ., \mathrm{qN}), \mathrm{q}^{1}$ belongs to $\mathrm{R}^{3}, \mathrm{i}=1, \ldots \mathrm{N}$; for any point $\mathrm{x}$ in $\mathrm{R}^{3}$ (the center of the collapse that will be defined next), define on the Hilbert space of the system the collapse operator

\section{$1 /\left(2 \pi \sigma^{2}\right)^{3 / 2}$ e $-(\mathrm{Qi}-\mathrm{x})^{2} / 2 \sigma^{2}$}

where Qi is the position operator of "particle" i. Here $\sigma$ is a new constant of nature of order of $10-7 \mathrm{~m}$. Let $\psi \mathrm{di}$ t0 be the initial wave function, i.e. The normalized wave function at some time t0 arbitrarily chosen as initial time. Then $\psi$ develops in the following way:

- It evolves unitarily, according to the Schrodinger's equation, until a random time $\mathrm{T} 1=\mathrm{t} 0+\Delta$ $\mathrm{T} 1$, so that

$\psi \mathrm{T} 1=\mathrm{U} \Delta \mathrm{t} 1 \psi \mathrm{t}^{\mathrm{o}}$,

where $\mathrm{Ut}$ is the unitary operator $\mathrm{Ut}=\mathrm{e}-1 / \mathrm{H}$ for every operator of $\mathrm{Ht}$, corrisponding to the standard Hamiltonian $\mathrm{H}$ governing the system, e.g. Given by (3) for $\mathrm{N}$ spinless particles, and $\Delta \mathrm{T} 1$ is a random time distributed according to the exponential distribution with rate $\mathrm{N} \lambda$ (where the quantity $\lambda$ is another constant of nature of the theory ${ }^{2}$, in order of 10-15 s-1.

- At time T1 it undergoes an instantaneous collapse with random center XI and random label II according to

$\psi T 1 I I$

$$
\psi T 1 \rightarrow \psi T 1+=\Lambda i 1(X 1)^{1 / 2} \Psi t 1 / I I \Lambda I 1(X 1)^{1 / 2}
$$

I1 is chosen at random in the set $(1, \ldots N)$ with uniform distribution. The center of the collapse X1 is chosen randomly with probability distribution ${ }^{3}$.

$$
P(X 1 \rightarrow d x 1 \rightarrow \psi T 1, I 1=i 1)
$$

- At the random time, the exponential distribution changes independently in wave function transformation.

The term probabilistic presents in three philosophic attributes: gnoseologic, ethical and ontic or physical. The physical model takes reference to:

- The probabilisme of the subatomic elementary matter of which occupies the quantistic mechanics
- The probabilisme of the macromolecular matter in terms of complexity

In probabilistic terms:

- In respect of the object the mental representation is true or false, as regards of the knowing subject it "appears" true or false, in probability and statistics ${ }^{27}$

- The number and the complexity of the connexions of a representation, will be it mathematical or physical, constitute the measurement criterion of a reliability since "persuasive and not-contraddictory" because it take part of the logics of physics ${ }^{28}$.

- Have the reinforcement of the "prevailement not contradictority" when the result was obtained in a method analytic and methodic, either from the grammatical point of view or that cognitive, otherwise through a method of inquiry correct and rational.

In probability the calculation's method take part of the analysis of complex systems, with it intends that has to obtain a reliable result, it can treat of an internal analysis, and it can be physically possible in respect of every possible further analysis. The contemporary probabilisme represents the "sphere" of the physical, otherwise of the matter in their being subject to the physics laws, when it is not deterministic. In the contemporary philosophy the probabilisme is the address of gnoseologic and scientific type for which the character of probability has recognized to some number of cases, subjected to indeterminism's shapes. Such systems regard the possibility to translate themselves in mechanic, physical and elaboration systems, and then of not - linear hypothesis as regards of the mechanics.

In the simple elementary systems ${ }^{29}$, the first to see indistinctly the probabilistic character of the physical reality was been Boltzmann, who had been yet understanding that the world of the physical elementariness would be not never to be intelligible in the physical laws of the microscopy, subjected to the classical mechanics. In refer to the simple physical systems, the probabilisme relates to especially the objects of the subatomic world, the elementary particles, inquired from the quantistic mechanics and some fundamental aspects of the standard model. So, through equi-probable measures we find an instrument 
of measurement of the physical laws that allow to classify or to ordinate the model of the elementary matter through a calculation of statistic type, otherwise of ordinal type. The complex macroscopic systems, in statistical logics, are physical systems that can involve in not-equilibrated states, or descriptive structures (so called by Prigogine, Nobel's prize of the 1977), such to determine some evolutions along new and different equilibriums more or less probable ${ }^{30}$.

The physics of continuum, in that data and metadatas are extracted in inverse formula between statistics mechanics and electromagnetism, does not allow a total classification of the chemical opposites, which in phenomenology they are considered a priori, excluding ones phenomenon of interference but only at the level of the entities of the particles. The physics, then, that extends all these phenomenon, admits ones exceptions only as regards to the hypothesis of the physics of the discret entities, as in a logic system with double entrance: the Thomson's theory in the physics of the quantities, being founded the physics of continuum by physic models, that is valid in this case, if it is associate to a partition physics an opposite theory, as that formulated by Rutherford for the sphere's circularity, in the electromagnetism, the system has to work as a function or as a breadth, or else still as a translation between the first atomic models and the informatics models. In fact, every survey is static for every distance, according to the phenomenon that replays measuring the difference. This phenomenon has been explained in many theories in general relativity, but some key-concepts can be founded again in the Newtonian physics. The statistic dispersion relationships foreseen by Popper do not coincide in the Rutherford planetary model, but however they take part of the quantisation in theoric physics though to a remarkable process from the statistics point of view. It is necessary to observe that, if the mechanics admits ones formulations of the density's field, they exist very extended relationships that have to be translated in polynomials of the statistic-data ${ }^{31}$. They are predicted, then, differential methods for the measurement of the data stastistics, the first executes a cognition for the frequencies, that take part of an elementary system or alternatively a system of first degree, the second method has to indicate some precision's rules of the measurement ${ }^{32}$, the third method is a complementarity of mathematical standards ${ }^{33}$, the fourth method is useful to verify of the statistics-data, in comparison with executed data in mathematics and in the logics of individualisation of data, the criterion to effect a division of $\mathrm{it}^{34}$. The problem, obviously, is to derive from it the object in that has to consist for the wave function that, taking part of the undulatory mechanics, would be statistically valid in the system of parameters determination, and as to the mechanics of the transitive mechanics ${ }^{35}$, variables in similar functions, the dimension allows to develop synthetically a problem of mechanics and physics though a comparison of the aggregated analysis data ${ }^{36}$.

The scientific interpretation allows to ordinate the mathematics and the philosophy though general phenomenon, that they are examined in logics, especially from the speculative grammar, from the logic critics and from the metodeutics, that being done by the same kind in terrestrial physics (of physical and chemical nature), is analogous to the special sciences and psychical sciences, in metaphysics, this is a theoric and practical application, and it is a method of the deduction and induction. This allows the classification and the analysis in linguistic criterions ${ }^{37}$, and it is very important to obtain a terminology ${ }^{38}$. From a philosophical point of view, the results obtained by Peirce and Cantor individuate, from my advise, a necessary classification, even if not sufficient, to describe the absolute infinity that avoids to the logicmathematical understanding of the set theory. Both describe such affinity as that exceeds the thought: in Peirce on discuss of a surplus of semiotic nature, that is to say of a reality that can be not defined with the same signs whose that identified in a set theory is described, whilst in Cantor on describe an excess of genuine metaphysic nature. Both of them makes in light the characteristics of exceedance.

It seems that it could be individuated as the characteristic element of a certain position that I will call "methodological metaphysical realism". As it is done, the excess does is not sufficient to define this position, nevertheless I will call methodological metaphysical realism a philosophical position only if it contain this type of element. The "methodological metaphysical realism" is defined by the demonstration of such "excess" of nature: both affirm the infinity's reality whom they speak about through a proof for absurd that individuates the limit of the concepts of the human being. In other words, both utilize the antinomies with the purpose to show the definition provided by a certain concept and all the possible operations on it do not exhaust the totality of the real and such conclusion is demonstrating perfectly by the thought. On have, then, the demonstration of the excess of the real through a method that the scholastics would have defined "for negative way". On keep that this do 
not mean at all that they must be there external implications, although in Cantor they would be evident, rather on obtain an original manner to consider the relationship between "physics" and "metaphysics", a way based on the reliability of the physics and the continuity between the one and the other reality, a continuity that has its hub or his contact point in the limits that the analytical thought meets when it thinks the totality and perhaps, more in general, that it meets every time it arrives to the extremity of what it have in some way to define, analyze and calculate. It is an interesting definition because it opposes as much to every ontologism's shape that finishes to separate or to oppose the two reality types so many to every shape of spiritualistic or materialistic monism, that holds out always to reduce the two types of reality to only one element. Neither Peirce or Cantor never sustained a similar philosophical position and, from my advise, in their philosophical studies they fallen down exactly to the internal of the two positions just described: Cantor declared himself openly to be a "platonic" so as Peirce considered himself an "objective idealist". They were definitions that picked in the sign because they were referred to the metaphysical considerations whom they reached even in the basis of own mathematical researches. From the mechanical data of informatics programming and calculation in series of numbers, it is necessary to establish a priori some casual variables, some models and formatting types in parallel (between series of proportions) and some indexes of ratio (on behalf to the choice of information's type). From the space analysis, from an analysis of geometric kind, in a problem of how to resolve a problem input/output to more variables, we introduce the Fermat's theorem. Leading from a calculation methodology that meets many static variables, for a demonstration of linear type, on treat in mathematical problem of how to resolve in a system classification a problems calculation of sequential type, in numeric integration. Beginning from the parallels and from the mathematical demonstrations relatives to the conversion of numeric data, and to the operation of reelaboration of curves, they are necessary the relationships. "Beginning from the 1970 or so, a series of mathematicians began to see indistinctly a strange nexus between the elliptic curves and the last Fermat's theorem simplifying, if Fermat had been wrong and then they had been existing two n-esime powers which sum is another n-esime power, for that these three numbers will have seen determining an elliptic curve. And if the sum of powers can be that, the elliptic curve will be very strange, with a surprising properties combination. So surprisingly, in effect, that seemed enormously improbable that could exist, as G. Frey observed in the 1985. This observation opens the way to a "demonstration for absurd", that Euclid called reduction ad absurdum. To demonstrate that an affirmation is true, it begins with the assumption that it will be, on the contrary, false. After on deduct the logic consequences of these falsities. If the consequences contradict between them, or they contradict any other known fact, then the starting assumption had been wrong, and for this the original affirmation is effectively true. In the $1980 \mathrm{~K}$. Ribet taken in point this idea demonstrating if the last Fermat's theorem is wrong, then the associated ellittic curve violates a conjecture (that is a possible theorem but not dimostrated) introduced by the Japanese mathematicians V. Taniyama and G. Shimura. This conjecture of Taniyama-Shimura, that goes back to the 1955 says that every elliptic curve is associated to a special class of elliptic function, called modular functions". It can be probable, in fact, to demonstrate that they exist numbers theories that develop angular implementation functions, done that in complex analysis it avail itself of geometrical projections. That are regressive on behalf to the flat. What does involves this projection? The existence, in fact, of a frequency, otherwise of an hyper-flat function, shows the necessity to dispose the numeric data through a relative frequency in similar way to a formatting. The Kepler's symmetry constitutes, on the other hand, a method of introduction for variables, and it suggests how to dispose the same variables in a complex system, if after to be affirmed some classes of numbers, obviously, an analysis of integral, the relationship between the numeric series and pre-established series obtains however a demonstration of how to determine a measurement instrument, in trigonometry, or some statistic-tests, that use the increase logarithms. The Lorenz's calculations, do not allow in every case to obtain geometrical representations of the type of information that will be at the same time of description. At this point, then, adopting a numeric frequency with the Benford theoric model, for example, it is possible to obtain some indicators of curve analysis, then, if the curve existence indicates problems of consecutive kind, with a valency, or a degree, in a point, the approximated calculation of the numeric series preestablished and analyzed in variable geometry in data, must be resolved necessarily with the analysis of the relative frequencies. The Riemann's analysis includes different types of solution to the approximating calculation of data in series, having in fact a first type 
of casual formatting and the second type that have to be calculated in quantitative analysis, they can be utilized the classical statistic hypothesis of Riemann, otherwise systems of inverse analysis for the type of information that use either the first or the second degree of algebraic analysis. The type of bivariate information re-enter in a finite algorithm of analogous systems, that allows a conditional analysis of the numeric succession method. "Generally speaking, we have the impression that a curve would be more "thin", for example, at the internal of a square. For too much time, the mathematicians have been considering that, seen that a curve is unidimesional and a square is bidimensional, will be improbable that a curve was passing for every internal point of a square. It is not so. In the 1980 the Italian mathematician G. Peano discovered a curve that fill up the flat. It was having an infinite length and an infinite number of creeks, but it re-entered still in the mathematical concept of curve, that in substance is a type of line more or less withy. The following year, the German mathematician D. Hilbert discovered another one. They are too complicated to be traced out, and even if it will be possible succeed in that after all it is worth to draw, a square totally dark, as the figure of a flat. The mathematicians define the curves that fill up the flat using a proceeding composed by following steps that add the creeks via via. To every step the creeks added are more small of the preceding. The illustration of the parallels [in approximation of the power of a number, ndr] shows the fifth step of this proceeding for the Hilbert's wave. At this point, the algebraic number theory is confirmed in the parts of the whole, meanwhile other options of this variable geometry, are the resulting of other frequencies, as for example, in the problem $\mathrm{P}=\mathrm{NP}$ in informatics ${ }^{39}$. The Fermat's theorem, relatively to a configuration of data, or to a super-programming, has a validity and an explicit construction, in the moment in which it had to be described in terms of classical physics" $"$.

\section{SECOND CHAPTER: MODELS TO SEPARATE THE VARIABLES AND THE SECOND ELABORATION}

The space is not an empirical concept abstract of the sensations. In fact, in order that some sensations have been referring to whatever out of me (that is something in a place of the space different from that I find myself) and likewise in order that I have been representing to me such sensations the one out and nearby to the other, and for that not only different, but in different places, it must be yet in foundation here the representation of the space". We observe: a) Kant confuses the sensation with the heard: they are the heard and not yet the sensations (the hear) these are referred to something out of me. B) The term out of me is ambiguous: it can be mean or simply separated from the I since acted to experience, from the act to hear, otherwise occupying a different place from that of my body. In the first meaning the term out of me is an immediate data and there is not any necessity of my space representation to have knowledge of it: also a pure sound that for itself, immediately, is not given to me as extended, is perceived as something of separated from me that $I$ hear. In the second meaning, the out of me is a notion to that it is possible to arrive after a certain repeated experience, it is not at all an immediate data, a property given immediately together with the heard, but it is an inferred property: the exteriority or the interiority of some objects to our body is learnt, and the learning process begins with the perception of the extended. [..] If after it would like to say that the exact measurement of the distances supposes the geometrical notion of space, it says a true thing; but the exact measurement of the distances is learn with a long process and it is not a presuppose of our perception of the extended, but, on the contrary, presuppose it.

4. "The space is represented as a given infinite greatness". E. Kant continues observing that every concept is potentially infinite, but any object contains actually in itself an infinite multiplicity. We observe that a spatial infinite is never given and that the representation of a space actually infinite is born from the contamination between the (potential) infinity of the space concept and the actual existence of a given concrete extensions, but this contamination will be bring in our fantasy and it does not authorize at all to affirm as existing the product of such determination"”.

5. It is affirmed the empirical data of a certain spatial infinity that is instead the "thesis", and the argument, that have been demonstrating the antithesis is deprived of value because it presupposes that the world, if it will be limited, it have been limiting in the void space, meanwhile the extended world is limited not by the void space, that does not exist, as says rightly Kant, but in his shape, from his figure (concept's extension).

6. Mathematics is a deductive science, Russell when affirms such identity, means to say that: 1) that in foundation of mathematics they are the same axioms that are foundation of the logic, 2) that the 
mathematics proceeds deductively, 3) that it is not lied to the world of sensitive quality. To understand this affirmation 1) it is necessary to take present that often it means for logic principles the supreme principles of the being (as the principle of identity and notcontradiction) that are to the foundation of the ontology and the logics (2).

7. If the contemporary mathematical logics has improved the Aristotle's logics and has assumed a complicity such to extract projection data, it give after the brief notions of logic minor in scholastic terms and in Aristotle's current terms rather than in terms of symbolic logics because, for that the same symbolic logicians say, all the aristotelic-scholastic logics minor is still valid (to the difference, for example, of the aristotelic physics) and the modern logics is not otherwise that an exposition more defined and more rigorous of the theories yet noted to Aristotle and the scholastics, to whose had been added others modern theories. The symbolic logics stays well then to the aristotelic-scholastic logics not as the contemporary physics stays to the ancient physics, but rather as the contemporary geometry, exposed with rigorous axiomatic method, stays to the Euclid's physics. Still they are studied the Euclid's elements. The exposition of the logics is necessary to understand, among other things, the philosophic logics or knowledge's theory.

8. Now, for the mathematics and the physics they are required necessary and universal propositions (seeing that without such propositions there is not really science) and the necessary and universal propositions can not to refer simply experience data, since the experience says to me only: "the things that I saw till now stay so". Then the necessary and universal propositions must be known independently from the experience, that is they must be a priori. A priori in this sense: that the connection between the terms must be known independently from the experience".

The analytical probability presents unlikely analyzing aspects, above all for the undulatory aspects owing to the phenomenon connected to entanglement. Often still it is associated a wave function, and then it is not lead out that in molecular physics they have been existing particular systems of descriptive probability to calculate the state of a system. In a spheric geometry, in fact, the ray can not to vary, because it is necessary to take reference to the angular unit for the spheric unit. It seems that on behalf to the Huygens-Fresnel's principle have been representing some aspects of the undulatory theory that the statistic varies on behalf of the method of binomials. Calculating the emission points, they exist greater lengths in comparison with these produced from the calculation instruments.

The inferential proceedings in the Maxwell's theory take to remarkable mathematical conclusions and in chromo-dynamics that leave out the "void effect" deriving from two fundamental equations that appear unfounded. (3-4)

Two artificial systems, having a sequential relationship 1 to $\mathrm{N}$, are analyzed in the whole of the times and in the measurement's variables in a transaction period that remains in the same conversion relationship. Pioneer and Voyager for the mission of spatial exploration started in the 1972, the measurements rings transfer the finite states of a system and the state of the entrances remains unvariable at the varying of a transaction system. The behavior of a combinatory system allows, however, to the computer to sequenciate the internal states of a system, if the state's variables, on behalf to the mathematical model, are represented at finite states, that is in a finite number, otherwise at finite states (for example, a safe), if the state's variables are in infinite number (for example, the universe).

It remains to understand what would be the evolution of artificial systems relatively to the transmission period, and then, what times Pioneer 10 calculates as regards to Voyager. "The two cosmic reporters develop in faultless manner their work. Hundreds of photo $s$ and measurements overtake the Earth and reveal the secrets of these far planets: the very intense magnetic field of Jupiter and the new rings of Saturn are the discoveries most exciting. As it happens frequently, the widening of the knowledge brings even new enigmas, new questions to answer. It is born so, to explore at the bottom the external planets, one of most ambitious missions until today: the "grand tour" of the solar system on behalf to the two probes Voyager. If we want to do a comparison with the human missions, the Voyager is the project Apollo of the spatial probes. The two probes leave in fifteen days of distance the one from the other of the 1977. An uncommon astronomic coincidence, the alignment of planets will allow to the Voyagers to exploit the gravity forces of the celestial body visited to arrive to that following"41. The measurement problem of the physical constants represents a method of composition of causal variables, that regards in first place the geometry, in second place the mathematics, in the experimental method 
nevertheless it is impossible to obtain in first approximation from a deterministic system because it exist one compared method in that the same experimental method return analogical physical models. On the structure's concept, it is possible to affirm the condition in opposite sense. Also the angular measurement allows to explain phenomenon of variable's regression. "The study ever more deepened of the intimate structure of the matter was lead the scientists to put even other interrogatives to themselves. In the XIX century, the light has been describing as the effect of an electromagnetic wave. A. Einstein, in the 1905 , was the first to perceive by the intuition that the light could be described even as a whole of particles, the photons, each of them transfer a determined quantity of electromagnetic energy. In this way, it was possible to explain many phenomenon that the only undulatory theory was not able to justify. Both the theories were then true: the light has been involving either as a flow of particles (the light quantum or photons) or as an electromagnetic wave. Going over the reasoning again done for the light, the French physician Louis de Broglie proposed to consider two electrons not only as particles, but also as waves. This allowed about it a complex mathematical treatment, as "wave functions" 42 .

In the research of an only one model of physical importance however, we find ourselves in spite of an hypothesis of uncertainty done by the linear system, and how this linear system could be translated in a static system. We have, then, in physics, physical and abstract models. The first are classic and analogic, the abstract physical systems are composed by graphic mathematical and logic models. If the logic and mathematical systems follow more than a direction, do not exist an attitude to commutation, for example, for the analogic systems. For this, the mathematical problems are descriptive and formals, or descriptive, synthetic and graphic. The problem to effect a computation on a string, for the Turing's machine, provides the alternating of a basis of data, and of a division of the basis of data. Given that the algorithm takes part in both the chances of the basis of data, will have even a computational basis, but the problem remains how to assume the properties of the organized machine, as for an "analytic machine". Newman utilizes a coherent system but the internal system of the not-organized machine does it allow to assembly basis of data through some data produced by the same calculator? The answer is yes, obviously, because we will have a configuration of the problem to effect a computational basis, and other binary configurations, the Hilbert's program, for example, separated a priori parts of the mathematics where it would be foreseen that an analysis of an infinitesimal calculation has not existed. This problem has been confronted by Frege and Russell, for the geometry of some complex figures, in analogous way to the Goldbach's conjecture, for which every positive whole number of two is the sum of two first numbers ${ }^{43}$. In the compilation process, the syntactic analyzer, or parsec, allows to configure an exact data on behalf to a casual series, of the analyzer, the syntactic analysis is a partition argument of first level of the lexical analysis, using systems of first degree to an unknown quantity of the analyzer, the compilation language does allow not a system transaction, for example, through some connectives (if/else/not). This is better represented as a terminal, that can define the BNF, that is the compilation's process syntax ${ }^{44}$. Between QCD (quantum chromodynamics) and the QED, the first in the description of the interaction between quark and gluons, the second through the field's interference, are described mechanical phases, that we can study though the geometrical optics and the undulatory optics. The computational physics, after, being placed for the same system of numeric analysis, provides different computational systems though mechanical phases in normal conditions ${ }^{45}$. The problem of first numbers and the Yang-Mills theory, so as the mass gap hypothesis are analyzed though particular functions on mathematics and physics. In spite of the problem of how to make possible a logics of first order between the two theories, in computational physics functions of computational complexity of different degree are provided and they are established from processes in exponential time* that are the theoretical parts of the complex systems, and then, there two theories put together in the "symmetry group" a new branch of mathematics, that is to say the groups theory. If it exist, in fact, a field's theory not quantum, as that of Maxwell, and if the Dirac's conclusion exhibits though a statistic set of samples the possibility to find a physical data in the symmetry's group, the QFT (the quantum field's theory) describes more than a wave function and if it is relative to an only electron, for example, otherwise to a statistic data given back though a basic set of sample in terms of particles. The wave's function, for example, is the inverse in proportions as regards to the Hamiltonian's operator, if the vector's shape of the Navier- Stokes ${ }^{46}$ equation, includes an integral as part of the equation. In fact, Feynman sustained an only manner to understand the 
mathematics though the quantum theory, but if this theory is associated to a linear algebras, or to a quantum physics of the QFL (theory of fourth level), it puts the problem in the mathematical theory to optimize in algebraic parts, as in the quantum chromodynamics (QCD), while if the physical information is divided, they exist different mathematical interpretations, that in probability are not provided by the Bayes methods, that found always the inverse or the proportional measurement of the Plank's report. They exist then different reports of a measurement of the wave's function, that are studied in physics, in chemistry and in logics as regards to the reports, the multiple-choice models, for whose a minimal function exists always to calculate the physical greatness of the phenomenon, the report of the information's transmission, and the invariance of the transformation, and if that transformation in the system is caused from two or more types of data, and how the process varies though fundamental arithmetic passages. The model for algorithms, though the formal logics, do not allows to resolve parts of the mathematical system, we have then the series, the vectorial functions and the continue functions ${ }^{47}$.Given a sequential model, they exist parts of the system that take part of the internal elements, the entrance's variables, these are the solicitations that can be varied (at least inside certain limits) by the intervention of the external environment (they are the so-called manipulating greatnesses) and the transictions or not-linear informations, commonly called disorder, which compose greatnesses that vary indipendently from every system control (called notmanipulating greatnesses), it refers to greatnesses that isolate the component of the system in spite of the final state of the function, that is assumed as calculation's function, and then of the same system, that measures the capacity of the calculator to assume two or more values in the transiction's system. The sequential model allows a transiction of system, but in order that it would operate as a static system, when the greatness does not continue in a continous function (the analogy permits to understand that static system and deterministic system allow linear variables, that are the opposite of the not-manipulating greatnesses), it must to allow a big variables number, but if to that system on add statistic greatnesses, the model become deterministic $^{48}$. In fact, in the choice of a computer, it happens however the state's transiction, because they are associated static, continuous and sequential models, beyond to constitute linear models for the calculation's process and the elaboration of inputs, because it represents a reduced system, then, it is as an equation system, but the same mathematical functions are to be resolved whether from the calculator or from the computer. This is resolved though a calculator in inverse processor, a system of inverse numeration, a geometrical description of the system $^{49}$. The numeration system does not allow a priori that the system could be varied in physical conditions, because it is a model in finite states, in fact, the physical models develop parithetic systems or analogic models, assuming the configuration, in the mostly cases, of static and directional models $\mathrm{s}^{50}$.

\section{THIRD CHAPTER: ELABORATION OF MODELS IN GEOMETRY UTILIZING CONNECTIVES AND OTHER MATHEMATICAL INSTRUMENTS}

The field of the analytic geometry belongs to the notEuclidean geometries for their influence on the concept of space, in fact, the deterministic systems, so as the expert systems, are utilized in analytic mechanics ${ }^{51}$. After the Euclidean geometries, usually, on take the problem of the exhaustion's problem, and the geometry of undividatings ${ }^{52}$, that is in this way necessary for the logics. In fact, in accordance with a relative classification, the logical calculation is structured according to different typologies, that correspond to development systems, which are the studies that try to create new informatic "environments" able to develop the systems of IA of the first three types. In substance, the flat develops on two different levels of logical analysis, as the propositional logics, the predicative logics and logics of greater order. In this case, it is necessary a demonstration as in the systems of elaboration of the natural language (Natural language Processing), indicated with the initials NLP; they are that whole of studies intended to build programs that would allow to the "machine" to understand an human language. Alternatively, the expert systems allow to the machine to make programming languages more direct, in the process of analysis of the calculator. It regards, however, of applications of the systems of elaboration. For example, it can be assumed to have three equations, as in the Newton's applications to the problem of tangents, of which one is formally is one component of that and takes part of the same tangent. The problem is composed of the area subtended of the curve, through a geometrical calculation ${ }^{53}$. It regards, however, of analogous processes to calculation of ponderable greatnesses, in similar way to the necessary calculation in the chemistry that occupies of the numeric calculations to the practical application of the 
rules and the laws regarding the substances composition and the reactions to whom they participate, or reactions mathematics, in which we must first of all to express the balanced equation of the reaction before to achieve an information ${ }^{54}$. It is possible also to represent the Lewis formulation through the valency's level, when charges are assigned to some atoms, they are called formal charges. These calculations in chemistry take part of the expert systems. From the point of view of the symmetry's analysis, we take referentiation however to an analysis of the static or dynamic variables or the systems of equivalence, otherwise of the symmetry of gauge ${ }^{55}$. They exist also some segmentations that allow to determine the probabilistic barriers existing in front of the computational physics, otherwise, if it regards deterministic barriers, it must to trait of calculating an exact value for the function of output in a computer. Otherwise, we can speak about transfinite computability also, and then on the possibility of a mathematical order at the internal of the system that utilizes the function of partition of the non-linear variables. From the qualitative point of view, we have two divisions: the autonomous systems and the complementary systems. The computational physics develops on dual criterions, on the Boolean logics and on the identification of half-wholes (or still, opposite values) ${ }^{56}$. The autonomous systems develops then on the grounds of the algebraic calculation, make as limit the greatness of the computer. The complementary systems assume as basis the main argument of the algorithm, make as an assumption of a mathematical calculation in machine language. The mechanicism of these calculator machines in comparison with the machines based on the value waited for (autopoiesis) presents informative transactional aspects that are whether from the statistical point of view or from the algebraic point of view considered finalistic. The synthesis is that the building of the physical outlook withdraws a microsystem through a machine with aspects very similar to the environment of distributed calculation $^{57}$. From this one can understand, through axiomatic and deductive character, that the "mathematism" of Descartes is a very modern method, because it is among the precursors of the complement's methodology in the complex ${ }^{58}$. "The Turing's solution is therefore applied and it is born from the more waste interpretation of the concept of machine with memorized program: the instructions for the management of the subroutine have been constituting a meta-program that was been applying to the main program, controlling times and addressing of the instructions. The strategy was based on the fact that the instructions were written in numeric code and could be elaborated as they would be data". With the aim to compose a $\lambda$-calculation the functional programming at the basis of that structured, is based on the development of the mathematics of Lagrange. The fixed TS of which Turing explains, at the basis of the process of computational accumulating and the informatics retrieval, is constituted by instructions directly tied to the problems of calculation of the register and the memory functions of the calculator, for this it obviously is that the $\lambda$-calculation is an algebraic mathematics and it is necessary to recur to the axioms as these of Poincaré, for example, for that it is easy to distinguish between structural programmation and not structural programmation. The differential analyzer, typical of the ENIAC machines for the organization of the machines and the methods of programming, represents a symbol of commutability, present ever in mechanics "to the implementation of the electronic machine they could be utilized the tubes at void (they were almost 18.000 tubes), developed to be used in analogical way in the radiophonic and telephonic communications since the years 30 . Also the flip-flop circuits (dual stable circuits, that have only two states of equilibrium), were used in great quantities in the ENIAC, although not still too long-winded. The true brave innovation of Eckert and Mauckly then was not in the architecture of the machine but in the trustful bet to take together industrial quantities of electronic circuits, convinced that they would have operated maintaining acceptable the level of the spoilts". The programmation's methods then represent a standard, constituted from positive vectors for alphanumbers and negative vectors for electronics (or by the programmation), for that is always better to isolate a certain kind of circuit. The process, then, at memorized programming is a process of transistor, in an appropriate way representable from a line of delay at the quicksilver.

To the mathematical operations at the basis are summarized proceedings systems in the binary calculation based on logical operations (A\&B, AvB, $<\mathrm{OR}$ ), appurtenance to $\mathrm{A}$ (and $\mathrm{A}=\mathrm{B}$ ), always unrolled on two TS fixed with a binary result (on a TS defined), on the grounds of the arithmetic part (CA) of the translations in machine language whilst the operation of transfer of the memory to the schedules utilized for the output and the input and vice versa, basing on the synchronization, are translated in the same way in which the machines non-organized are based on the 
elaboration and the dual commutation. "One can speak about it in the words of a physicalism and philosophical mechanicism and physicalist mechanicism $[\ldots]^{59}$. The unconditional jump instead did not utilize a special register to re-address the instructions, but was governed directly from a succession of instructions that have been modifying other instructions. The adopted technics was maybe not necessarily complex, but we notice that Turing adopting this proceedings has been leading to the extreme consequences the notion of memorized program". The partition's work concludes almost always with an indefinite integration. To this problem, usually, on applicate a mechanical proceeding in which they would be present relationships and quantifiers to allow the complement's function ${ }^{60}$. At this point the planning out can be physical or mathematical: if on assume the one physical, then on take two samples, and the unit, otherwise, the division is measured though a quantifier, if it regards that mathematical, we have different choices. But usually that presenting two values in one is that preferring. The system of the classes at this point is divided by a partition. If they are identified determined elements, then we will have a mathematical equation.

The Descates's method is equivalent to allow in geometry the utilization of algebraic curves of whatever degree, that can be classified on the grounds of their degree: for example, the straight line is at the first degree, the conic sections at the second, the cissoids at third and the concoid at fourth. And Newton showed that the utilization of the alone concoid allows to resolve all the problems that can be translated in equations until the fourth degree: in particular, all the ones that were resolvable though squared line and compasses collapsible or though conic sections. But also the Cartesian geometry had serious limitations, because the algebraic representation of a curve does not mirror again its geometrical complexity. For example, the equation of a parabole $\left(y=a^{2}\right)$ is more simple than that of a circle $\left(x^{2}+y^{2}=r^{2}\right)$, but a circle is more easily be built of a parabole. Otherwise, curves as the spiral of Archimedes or the cycloid of Galileo are easy to generate mathematically, but impossible to express algebraicly. Newton proposed then to achieve the last stride and to arrive to the analytical geometry, that allows the utilization of whatever analytical curves, and justified it as the natural extension of the classical geometry though processes at the limit. For example, since $1 / 3$ is equal to the summarization of the infinite series $1 / 2$ less $1 / 4$ more $1 / 8$ less $1 / 16$, and so-on, the tri- section of the angle became simply the limit of an infinite series of subsequent bisections. The utilization of analytical curves as the spiral of Archimedes or the cycloid of Galileo allows to resolve insoluble problems through algebraic methods, as the famous circle's quadrature. A problem that, on impressed, can be easily resolved also with the Egyptian methods, keeping unitary the circumference and building a square of side equal to the squared root of its half (think that can be done with line and compasses)" " "On the one hand, the electronic instruments are more useful when on traits to answer to quantitative questions. Electronic revealers as the counter Geiger, that measures the radioactivity in the cellars of old houses, are founded on the logics. They are programmed in way to answer to simple questions every time that reveal a particle, and to register if the answers to the questions are yes or not. They can discover collisions of particles to frequencies of millions to the second, to subdivide them in yes and not, and to count the number of answers yes and the number of answers not. The history of particle's physics can be divided in two periods: in the first, that finished around the 1980, optical revealers and optical images were determinant, whilst in the second electronic revealers and the logic took over. Before this transaction, the science was advanced making qualitative discovers of new particles and new relationships between particles"62. "The theoric science can be divided roughly in two parts: analytic and synthetic. The analytic science reduces complex phenomenon to their components most simple. The synthetic science builds complicated structures beginning from their most simple parts. [...] Another reason for that I believe that the science would be inexhaustible is the Gödel's theorem. The mathematician Kurt Gödel discovered and demonstrated his theorem in the 1931. The theorem says that, given a whatever finite series of rules to make mathematics, there are undecidable propositions, that is to say mathematical propositions that can be not neither demonstrated or disproved utilizing such rules. Gödel furnished examples of undecidable propositions that can be not demonstrated neither true of false using the normal rules of the logics and the mathematics. His theorem involves that the pure mathematics would be inexhaustible. For all those problems we can resolve, there will be always others that will be not resolved with the existent rules. Now I sustain that, in consequence of the Gödel's theorem, the physics also is inexhaustible ${ }^{63}$. The physics laws are a finite whole of rules, and they belong the rules to make mathematics, so that the Gödel's theorem applies ever 
to them. The theorem implicates that ever inside the fields of equations of basis of physics ${ }^{64}$, our knowledge will be always incomplete". "While the notion of such transfinite computations is perfectly coherent, whether or not an $\alpha$-Turing machine is physically realizable for a given ordinal $\alpha$ depends on what physical theory one takes to characterize physical possibility. An $\alpha \times \alpha$ partition of space-time into computational steps and tape positions is realizable in a space-time constructed from $\mathrm{R}$ if and only if $\alpha$ is computable. On the other hand, the $\omega 1$-Turing machine, where $\omega 1$ is the first uncountable ordinal, is in principle realizable in a nonstandard space-time, constructed from the hyperreal (the nonstandard reals of nonstandard analysis) $* \mathrm{R}$ or from the long line $\omega 1 \times[0,1)$, since both spaces allow for uncountable ordered partitions. That is, what computations are physically possible depends in part on the topological properties of space-time. In order to see this more clearly, we begin with the theorem just mentioned concerning order-preserving injections into the reals.

Theorem 1. (Real injection Theorem). There is an order-preserving injection of an ordinal $\alpha$ into $R$ if and only if a is countable."

"Similarly, an $\alpha$ partition of space that represents an ordered tape is possible if and only if there is an orderpreserving injection from $\alpha$ to the parameter representing physical tape locations".

"Theorem 2. (Hyperreal Injection Theorem). There is an order-preserving injection from the first uncountable ordinal $\omega 1$ into the hyperreal (the nonstandard line ) *R."

"One should distinguish symmetries of a set of situations to which a theory may be applied from symmetries internal to that theory. One place to look for theoretical symmetries in a dynamical theory of physics is in its equations of motion. Since these equations pick out a class of dynamically possible models, one can alternatively focus on symmetries of this class of models. It is not necessary to endorse any version of the so-called semantic conception of scientific theories to acknowledge that many physical theories, as well as theories in other sciences, are often conveniently characterized by specifying the class of models associated with the theory. Here models are structures (typically mathematical) that may be used to represent situations. So an analysis of a theoretical symmetry as a transformation that maps models of a theory on to other models may be expected to be widely applicable. But what kind of transformation? On the broadest conception, a theoretical symmetry would be any 1-1 function from the set of a theory's models on to itself. But while this is a symmetry of the theory in the sense that it leaves its model class invariant, it is too broad to be of much interest. As Ismael and van Frassen [2003] noted, there are theoretical symmetries in this sense that transform a model of Newton's theory with one free particle into models with millions of particles interacting in complex ways. As an automorphism of the model class of a theory, an interesting theoretical symmetry should preserve more of the internal structure of the models it relates: cardinality of that structure 's domain is only one very weak requirement. Ismael and van Frassen [2003] entertain another condition: that a theoretical symmetry preserve qualitative features of every model. They take such features to be quantities that can characterize a situation, distinguishable by even a gross discrimination of color, texture, smell and so on.' (pag. 376), where (as they have explained) a quality can be regarded as a quantity with the range of values 1 (possessed) and 0 (not possessed). To maintain the present clear distinction between models and situations, one should rather characterize the qualitative features of a model as those elements of the model that may serve to represent qualitative features (in their sense) of situations.

They distinguish this condition from a stronger condition - that a theoretical symmetry preserve measurable features of a model, where these generally extend beyond qualitative features in a theory-guided way. Newtonian theory, for example, connects the masses and forces its models are intended to represent to qualitative features such as positions and times in such a way as to permit the measurement of the former by observation of the latter. In the case of spacetime theories, a theoretical symmetry may be required to preserve those features of its models that serve to represent spacetime structure, which give rise to the notion of a spacetime symmetry of a theory. So, for example, spacetime translations and rotations are spacetime symmetries of a Newtonian theory, while Galileian boosts are spacetime symmetries only that theory's models do not permit the representation of a privileged state of absolute rest."

"A substantial part of the mathematical researches of Newton regarded the so called "organic approach to the curves", where the adjective was intended in the greek sense of organon, "instrument". It regarded in other words to waste the tools box that allow to generate 
curves, placing side by side new instruments to the two classical of the lines and the compasses. Looking at the conic sections, since the antiquity various organic descriptions were known, for example that well noticed of the ellipse, with an hold out rope anchored to the two fires. Newton founded of it one new and powerful when he was young, in theirs admirable years; and he edited it in the 1687 in the Philosophiae Naturalis Principia Mathematica as Lemma i.21, applying it in the proposition i.22 to the problem to describe an orbit that would pass for five given points. The idea is simple. It is possible to take two couples of straightedges disposed at a fixed angle, and with the hinges anchored on two points: making to move the intersection of two corresponding straightedges along a given curve (the directrix), the intersection of the others two corresponding straightedges generates a new curve. If the directrix is a straight line, on obtain so all an only the conic sections. If the directrix is a conics, on obtain cubicals or quarticals. And, generally speaking, if the directrix is an algebraic curve on obtain curves of superior level on behalf on its own. In the same spirit, Newton founded an organic description of the cissoids of Diocles. On traits of the cubics described from the vertex of a parabole that rolls externally in his specular image, forming a curve "with shape of a polygon", that explains his greek name. Diocles was used it to resolve the problem of the duplication of the curve, and Newton generated it with a couple of straightedges with straight angle, one of them passes for a fixed buttonhole: whilst the extreme of the other moves along a straight line, his medium point describes the cissoids. The geometrical result most important and lasting of Newton regards the cubics in general; the classification of all their possible 78 types, of which few were known before him. He divided the cubics in four families, that after he divided in further sub-families. And he discovered that, as all the conic sections are sub-sections of a circle, so all the cubics are projections of five types of elliptic curves, that will be so called afterwards for the reason of their role in the calculation of the length of ellipse's archs (to avoid all the misunderstandings, the ellipse is not an elliptic curve). But Newton did not explain how he was obtained the results: probably anticipating the methods of the modern projective geometry. Cubics apart from this, his preferred curve was the concoid of Nicomedes, almost simple to build as a circle: it is described from the extreme of a straightedge passing for two buttonholes, one fixed and the other mobile along the straight line. Newton demonstrated that it is an universal quarticle, that it allows to resolve the problems bringing again to the equations until the fourth degree: included the trisection of the angle and the duplication of the cube. Among the not algebraic curves, he considered instead particularly natural the cycloid of Galileo, that is described from a point on the border of a wheel that rolls. Newton demonstrated that allows to divide an angle in a whatever number of parts: included three, obviously. And it allows to resolve the other classical greek problem of the quadrature of the circle. The cycloid is ever the solution of the famous problem of the brachistocrone, or curve of "maximum time" of route, throw in the 1696from Johann Bernoulli”.

"Guildin was attacked after the foundations of the Cavalieri's method: the notion that the flat would be composed from an infinity of straight lines, and a solid from an infinity of surfaces. This idea was persisted in Guildin, did not have any sense: "Any student of geometry will concede to him that the surface corresponds to "all the lines of such figure", and it could be called so in the geometric language". In other words, given that the lines do not have length, however much numerous they could be, one near to the other they would not cover neither the most small flat figure. The Cavalieri's attempt to calculate the area of a flat figure from the dimension of "all his lines" was then absurd. Guildin was arrived then its conclusive argumentation: the Cavalieri's method was based on the construction of the relation among all the lines of a figure and all these of another. But, sustained Guldin, both the wholes are infinite, and the relationship between two infinites does not have meaning. It isn't necessary how many times an infinite number of indivisibles can be multiplied, they will be not ever more numerous of another infinite whole of indivisibles".

Cavalieri [...] denies to have assumed that the continuous would be composed from an infinity of indivisible parts, explaining that his method does not depend from this point of departure. If it would be thought the continuous is composed from indivisibles, then yes, "all the lines" together effectively summarize to make a surface and "all the flats" to make a volume, but if it is not allowed that the lines compose a surface, then there in the middle there is undoubtedly something - besides the lines - that generates the surface and something besides the flats that generates the volume. Nothing of all this, he sustain, had to do with the indivisibles method, that compares all the lines or all the flats of a figure with these of another, 
independently from the fact if, in effect, they would compose the figure or not. The Cavalieri's experiments here could been technically acceptable, but they were also unsincere. Whoever would have read his book of 1635, Geometria Indivisibilibus or the Exercitationes would not have had doubts that they would be based on the fundamental intuition the continuous would be composed from indivisibles. Guildin had perfectly reason to ask about it to Cavalieri on his views on the continuous, and the defence of the Jesuit seems an excuse rather weak. The Cavalieri's answer to the Guildin persistence in the fact that "an infinite has not proportion or relationship with another infinite" was not roughly more convincing. He was distinguished between two types of infinite, sustaining that an "absolute infinite" effectively has not relationship with another "absolute infinite", but all the straight lines and all the flats have an infinitively not absolute but "relative". This kind of infinite, he continued, can be relation with another relative infinite, and it has it. As before, Cavalieri seemed to defence his method on an abstruse technical flat, that could be or not to be acceptable for the colleagues mathematicians. In every case, his arguments do not have any relationship with the true statement of motives that was concealed behind the method of the indivisibles" ${ }^{\text {. }}$.

Popper affirms then in the Logics of the social sciences and other essays:

- The problem's solution of how to interpreter the probability's theory is fundamental for the interpretation of the quantum's theory; the quantum's theory is, in fact, a probabilistic theory.

- The idea of a statistic interpretation is correct, but it misses of clearness

- As consequence of this lack of clearness the usual interpretation of the probability in physics oscillates between two extremes: an objectivistic interpretation, purely statistic and a subjectivistic interpretation, in terms of uncompleteness of our knowledge, or the information at disposal

- In the orthodox interpretation of the quantum's theory by the Copenhagen's school we find the same oscillation between an objectivistic interpretation and a subjectivistic interpretation: the famous interference of the observer in the physics

- In contraposition to all this, it comes here proposed a statistic interpretation, reviewed and corrected. It traits of the propensity's interpretation of the probability.

- The propensity's interpretation is an interpretation purely objectivistic. It eliminates the oscillation between the objectivistic interpretation and the subjectivistic, and with it the interference of the subject in the physics

- The idea of propensity is "metaphysical": exactly in the same sense in which in this manner are the forces and the fields of force

- It is also "metaphysical" in another sense: in the sense it provides a coherent program for the research in physics.

"I have underlined as the propensities would be not only objectives compared with the experiment's formalities, but also physically reals - in the sense in that they are physically reals the forces and the fields of forces - in spite of this they are not waves-pilot in the ordinary space, but functions-weight of possibilities, that is worth to say vectors in the space of possibilities (the Bohm's "potential quantum's mechanic" could to become in this context a propensity, to accelerate more than an accelerator force. They would give a certain weight to the Pauli-Einstein's critics of the theory of waves-pilot of de Broglie and Bohm". At this point, the relative division of the classical mathematics occupies to distinguish the categories on the grounds of the purely formal concept, otherwise to the mathematization, and then the classical geometry divided in Euclidean spaces at three dimensions involving the numeric analysis".* "An algebra consists of a non-empty set A together with some operations defined on A, each one with its arity. An n-ary operation on $\mathrm{A}$ is a function from $\mathrm{An}$ to $\mathrm{A}$, i.e. A map that assigns an element of $\mathrm{A}$ to each sequence $<\mathrm{a}^{1}, \ldots$ an $>$ of elements of A. A 0 -ary operation is just an element of A. Some common examples of algebras are groups, rings and lattices; some algebras related to logic are Boolean algebras (corresponding to classical logic). Heyting algebras (intuitionistic logic) and MValgebras (related to Lukasiewicz many-valued logic). 
Algebras are denoted by $\mathrm{A}=\left\langle\mathrm{A}, \mathrm{f}^{1} \mathrm{~m}, \mathrm{f}^{2} \mathrm{n}, \ldots>\right.$, where $\mathrm{A}$ is the under-lying set of universe of the algebra and $\mathrm{f}^{1} \mathrm{~m}, \mathrm{f}^{2} \mathrm{n}, \ldots$ are the algebraic operations with arity $\mathrm{m}, \mathrm{n}$. Usually we do not indicate the arity of the connectives, as the context suffices to determine it; on the other hand, we shall sometimes write fA when we want to stress that the operation $\mathrm{f}$ is defined on the algebra A. In order to speak about algebras we use an ordinary first-order language with equality (for which we use the symbol $\approx$ ) and without any other relation symbol; we use a function symbol of the appropriate arity to represent each operation of the algebra. We call this kind of language an algebraic language. Since we are interested in relating algebras to propositional logics, we consider algebraic languages that are built from the propositional ones in the following way. Given a propositional language $\mathrm{L}$, consisting of a set of variables plus a set of propositional connectives, we take any propositional variable $\mathrm{p} \varepsilon \mathrm{L}$ as a variable of the algebraic language, and any propositional connective of $\mathrm{L}$ as function symbol of the appropriate arity of the algebraic language. In this way we have that the propositional formulas of $\mathrm{L}$ coincide with the terms of the algebraic language. The formulas of the algebraic language are then built form terms in the usual way, for instance the atomic ones have the form $\varphi \approx \psi$, where $\varphi$ and $\psi$ are terms, i.e. formulas of the propositional language $\mathrm{L}$. This kind of atomic formula of the algebraic language is called an equation. Equations, as the are about to see, have a key role in algebraic logic; since they are pairs of formulas, we shall sometimes write $\langle\varphi, \psi\rangle$ to denote the equation $\varphi=\psi$. Another subclass that has special interest is that of quasi-equation. By a quasi-equation we mean a formula of the following form: $\left(E^{1}, \& \ldots \& E n\right) \rightarrow E$, where all $E^{i} i$ are equations and the connectives \& and $\rightarrow$ refer to the ordinary first order conjunction and implication. Note that any equation is also an quasiequation (an implication with empty antecedent $)^{66}$.The probability distribution regards certain dispositional properties of the field, that unrolls to its turn on more levels. This is anything else that the result of the relative properties to the experiment's predisposition. This is translated in quantitative linguistics in a particular idea of statistic law, otherwise in the superficial structure of the theory ${ }^{67}$. Analogously the Boltzmann's theories for the generative linguistics allow to understand that it traits of a possible identity on the flat of the geometry to the physics $^{68}$. It traits also of relations of dispersion that however find some relations in the system, as in the curves, otherwise in differential geometry, of which to speak about it seems strange in special relativity of at least they could be unrolled anyway adjunctive hypothesis on the curves of geometry from the point of view of in physics, generally speaking" 69 .

The second model of SAD computation is Hogarth's [1992], [1994], p. 126, [1996], pp. 91-4, [2004], pp. 681-2, [2009], pp. 281-3). The strategy is to find a relativistic spacetime structure (1) that allows a human user to survey the entire, infinite worldline of some finitary computer.

This requires a Malament-Hogarth spacetime, which is a time-oriented differentiable manifold with a Lorentz metric and three essential components (Hogarth [1992], p. 176):

$\gamma:$ A time-like path with a start-point but no end-point, such as $\int \gamma d \tau$ is infinite.

$\mathrm{R}$ : a point that contains $\gamma$ in its past.

$\mathrm{P}$ : a time-like path, from some point $\mathrm{q}$ to $\mathrm{r}$, such that $\int \mathrm{r}(\mathrm{q}, \mathrm{r}) \mathrm{d} \tau$ is finite.

[...] I treat the notion of physical computability as follows:

Physical computability: A function, f, is physically computable at a world if and only if there is a machine "blueprint" such that, for all $\mathrm{x}$, it is physically possible for there to be a machine instantiating that blueprint which physically outputs $\mathrm{f}(\mathrm{x})$ on input $\mathrm{x}$.

Understood in this way, the answer to the question "which functions are physically computable?" obviously depends upon which notion of physical possibility is in play. I will discuss the appropriate sense of physical possibility in some detail below.

The Physical Church-Turing Thesis states that the physically computable functions are exactly the Turing computable functions. So, if there is some sense in which it is possible to build a SAD computer, the Physical Church-Turing Thesis is false in that sense.

I have no desire to show that the Physical ChurchTuring Thesis is true, nor to determine what the physically computable functions are. I simply want to show how the Physical Church-Turing Thesis will have to be defended, given that we have described physical models of SAD computability. My discussion comes in two halves. In Section 2.2, I consider deterministic barriers to physical computations. In Section 2.3, I consider probabilistic barriers to physical computations.[...]

Accordingly, even if there is an actual deterministic upper barrier to Turing computability, the Physical 
Church-Turing Thesis may be defensible. We just need to find a legitimate sense of physical possibility such that

- for every physically possible world, there is some deterministic upper barrier to finite physical computation within that world.

- $\quad$ For every deterministic upper barrier to finite physical computation, there is a world that transcends that barrier. [...]

In particular, I shall focus on the following argument, which I think fairly summarizes Hogarth's attack on effectiveness:

(a) which functions can be computed by a (configuration of) physical Turing machine(s) depends upon the structure of spacetime.

(b) The structure of spacetime is either to be determined by physicists, or to be stipulated within some formal system; there is no third way.

- So which functions can be computed by a (configuration of) Turing machine(s) is either a purely formal or purely physical question; there are no interesting questions about effective computability.

The importance of finite computation becomes even more obvious when we consider Welch's [2008] completely general characterization of the hierarchy of SAD computers, as summarized above in Section 1.5. Condition (1) states that, at base, all the computation that take place in a $\mathrm{SAD} \alpha$ computer must be built up from finite computers. Furthermore, condition (3) ultimately entails that the tree-structure of any SAD $\alpha$ computer must be given by a (finite) recursive ordinary; that is, the structure of the computational tree must itself be finite computable. In short, SAD computation is built up from finite computation in finite computable steps. This all contrasts sharply with the case of geometry. Euclidean geometry is not built up from Lorentz geometry, and Lorentz's geometry is not built up from Euclidean geometry. On the contrary: these two geometric theories have mutually contradictory axiom concerning parallel lines and so have different theorems about circles (for example). By contrast, the axioms of the formal theory of SAD computability will contain all the axioms of the formal theory of Turing computability. So this is the point at which Hogarth's analogy with geometry collapses ${ }^{70}$.

In a system given for definition as physical on assume a relationship and that system, that assumes a role on a physical part of the empirical basis, containing some objective data, adopts a logics of the objective condition postponed to the formal logics, above all for the operators of the logic analysis ${ }^{71}$. When a relationship comes before these three properties, then it is of the type that gives an origin to an order between the terms for which it subsists, and whereas an order exists, it is possible to find always that this is generated from a relation that has these three properties. Before to illustrate this thesis, we will introduce some definitions:

- A relation is said a synonym or that involves difference or that must to be contained in when the term does not have this relation with himself. For example, "more big", "of different sides", "brother", "husband", "father" are aleo-relatives; they are not instead "equal", "born from the same parents", "dear friend" and so on.

- The square of a relation is that relation that is worth between two terms $\mathrm{x}$ and $\mathrm{z}$ when exists an intermediate term $y$ such that the relation given is worth between $\mathrm{x}$ and $\mathrm{y}$ and between $\mathrm{y}$ and z. Then "paternal grandfather" is the square of "father" and "more big than 2 " is the square of "more big than 1" and so on.

- The domain of a relationship is formed from all the terms that have the given relation with other terms, whilst the inverse dominion is constituted from all the terms with that the terms of before have the given relationship.

These expressions are yet been defined, but we have here to repeat them to establish the following definitions:

- The field of a relation is formed by the dominion and by the inverse dominion, taken together;

- A relationship involves or is implicated in another if it is worth when the other is worth.

It can be seen that an asymmetric relationship is a relationship which square is aleo-relative. It happens often that a relationship would be aleo-relative without to be asymmetric, but if an asymmetric relationship is always aleo-relative. For example, "spouse" is aleorelative, but it is asymmetric, because if $\mathrm{x}$ is spouse of $\mathrm{y}, \mathrm{y}$ is spouse of $\mathrm{x}$. For the transitive relationships still, all the aleo-relatives are asymmetric as it is true also the vice versa. From the definitions it can be seen that a 
transitive relationship is implicated in his square or, as we can also to say, it involves its square [..]. A relationship is connected when, given two whatever terms of its field, the relationship is worth between the first and the second or between the second and the first (not excluding the possibility that they would to succeed all the two thinks, even if this can happen not if the relationship is asymmetric) $)^{72}$.

For the controllability of data and metadata not in theory, the system in mathematical logics achieves flexions very more less in accordance with the controllability in formal theory, both it would be semantics, and organizational and technical"3. "We can then to proceed to general affirmations, as " $x R y$ is at times true", that is, they exist cases in that dual relationships are worth. A similar affirmation belongs to the dominion of the logics (or the mathematics) in the sense in that we have yet used the word. But we have to notice that in this affirmation it is not possible to make any sign of particular thinks or particular relationships. Particular thinks or particular relationships can enter not in a proposition of the pure logics. The only possible components of the logical propositions stay the pure shapes. I do not want to affirm decisively that the pure forms, as for example "xRy", would entry really in the propositions of the type we are to consider. The analysis of these propositions is a complicated problem, rich of contradictory considerations in a sense and in the other [..]. A characteristics then necessary (but not sufficient) of the propositions of the logics and the mathematics is that they must to be obtained from a proposition not containing variables (that is to say not words as "all", "some", "one", "the", etc.) changing every component of the proposition in a variable and affirming that the result is always true or at times true; otherwise that, in accordance with some variables, it is always true that the result is at times true in accordance with the others; otherwise a whatever variation of these shapes. Another way to express the same concept consists in to say the logics (or the mathematics) considers only the "shapes " and traits of them only to establish if they are always or at times true, with all the permutations of "always" and "at times" that one wants" "74. It traits, then, of assertions of the mathematical logics retained conceptually valid, but that take away from the main function for some properties. An approach to the systematic truth of particular logical functions seems to belong to the inferential statistics, whilst, for other methods, it takes part of the analytical philosophy. Russell, at this purpose, affirms: "the limit" for a given argument a exists only when all four of these limits are equal, and it is then their common value. If this is also the value of the function for the argument $a$, the function is, in that point, continue. This can be took as definition of continuity, being equivalent perfectly to the first given definition of continuity. We can define the limit (if it exists) of a function for a given argument without to pass for the case, properly as the first definition of continuity. We define before the limit from "left". For the existence of a left limit when on holds out to a, it is necessary and sufficient that, given a whatever small number $\sigma$, two values sufficiently near to a of the independent variable (both less of a), would be less different of $\sigma$ : in other words, if $\varepsilon$ is sufficiently small, and our arguments lie both between $\alpha-\varepsilon$ and a (excluded) then the difference between the values of the function for these arguments must be minor of $\sigma$. This must to be worth for whatever $\sigma$, how much small it would be; in such case the function owns a right limit. These two limits, also when they exist both, must be not identical by the force; if they are identical, they can to be not still the same to the value of the function for the argument a. A function is called "continue" without further modifications when it is continue for every argument. Another method, a lot different, to arrive to the definition of continuity is the following: we say that a function "converges finally in a class a" if a real number exists such that, for this argument and for all the greater arguments of this, the value of the function is member of the class a. Similarly we will say that a function "converges in a when an argument holds out to $\mathrm{x}$ from below" if an argument $y$ less of $x$ exists such that, in all the interval from $y$ (included) to a (excluded) the function has values that are members of a. We can now to say that a function is continuous for the argument a, for which it has the value $\mathrm{f}(\mathrm{a})$, if it satisfy four conditions, that is:

- Given a whatever real number smaller of $\mathrm{f}(\mathrm{a})$, the function converges in the successors of this number when the argument holds out to a from below.

- Given a whatever real number greater of $\mathrm{f}(\mathrm{a})$, the function converges in the predecessors of this number when the argument holds out to a from below.

$<3>$ and $<4>$ are conditions similar for when the variable holds out to a from upon. The advantage of this shape of definition consists in the fact that they are analyzed the conditions of continuity in four parts, 
considering arguments and values respectively greater and smaller of the arguments and the values for that the continuity must to be defined ${ }^{75}$.

\section{NOTES}

1. A. Blasi, Mathematics, exercises, complements and preliminary arguments, Publishers Kappa, 2004, p. 222-225

2. Philosophical encyclopedia Bompiani, voice logics of the predicates, p. 6574 .

3. Metaphysics for absurd, G. Maddalena, Peirce and the problems of the contemporary epistemology, Rubbettino, 2009, p. 141.

4. The deficiency of the Cantorian definitions, according to Peirce, is that they are not definitions, the "think many as one" is only a psychic experiment, a description more than a description. Instead the set theory demands a more exact definition of his fundamental term, that remains otherwise too much vague for to be utilizable. That the Peirce's observation would get in the sign, it suggest to me proposed even by the fact that the same Cantor in the quoted phrases had been looked for stating precisely own description of the "union in an all" or of "think the many as one", at first with the philosophical reference to Plato and, subsequently, with the adjectives "determined and well defined", applied to the objects and with the reference to particular faculties as the intuition and the thought. It is noted even in this second choice it is regarded as terms that have a strong philosophical connotation. In particular the reference to the faculties of intuition and thought, that serves to explain how it would come erring the relationship with the objects, and his grouping in sets (criterion of appurtenance), makes in light the implications of epistemology that burden on the same concept of set. It stands out so that the tie epistemology-set theory is the fundamental nexus of all the plant that develop itself beginning to the same concept of whole. Not casually, as Wittgenstein will give back as evident in the Tractatus, the refuse of epistemology will be often connected with that of the sets theory, intended as foundation of logics. Metaphysics for absurd, p. 170.

5. L. Boltzmann, Mathematical models, physics and philosophy, p. 142, argument expressed at p. 170 for the concept of number.
6. L. Boltzmann, Mathematical models, physics and philosophy, divulgative written, p. 46-47.

\section{Boltzmann, p. 173}

8. Descartes, Discourse on the method, note on Cassirer, p. 46, regarding the modern parallelism between mathematical truths and logic principles.

9. Descartes, Discourse on the method, p. 105 (sixth part) on the project of a computational machine extremely elevating.

9. 1. On the model of Kant, Leibniz and Locke. This model today utilizes the cardinality of the whole, though, for example, the diagonal argument of Cantor.

10. D. Shasha and C. Lazere, Computer to DNA, The future of intelligent machines, The Sciences, 2010, p. 182. "In molecular dynamics on exam the configurations of the poli-atomic structures, included the other atoms present in the surrounding environment, and after on determine in what way these configurations change in the time in answer to the forces that the atoms exercise the ones or the others. The proteins, typically, are done of thousands of atoms. Furthermore, there are the molecules of water surroundings. There is not "synthetic formula" that would foresee the courses, there is not a way to resolve the problem limiting to manipulate equations. The only thing is to recur to the simulations. To do a simulation of molecular dynamics, on divide the time in intervals extremely short, of the order of the femtosecond. A femtosecond is equal to 10-15 seconds, a thousandth of milliardesim of second. For each of these temporal intervals, on calculate the forces that exercise among all these atoms, and often is calculated the position in which have been moving each of them, in reason of these forces, in the following femtosecond. [..] Every request of calculation of the forces [it is first of all sequential, but it is necessary to leave from a simulation, that has to terminate with the calculation of the preceding in distance or in time's limit]. If the relative information to each atom is localized in one of the processors of the parallel machine, we can imagine rather a lot of approaches. The most natural would be to send the information relative to the atom A toward the positions of each of his near B. in the terminology adopted by Shaw, this means to send the information of A in the territory of each of his near B, symmetrically, A has to receive, in his territory, the relative information to each of B that has near. This schedule, therefore, requires still a great number of displacement 
of data. The Shaw's method utilize the geometrical configuration to realize that the passage of the information from A to B would take place in a "neuter" territory, neither in the point in which A finds or in that finds $\mathrm{B}$. the methods based on the neuter territory succeed in to accelerate the calculations for the conventional clusters of parallel processors, but Shaw is gone on the other side of the simple planning of algorithms, to build instead his parallel machine, that called Anton. (The name derives from that of Antoni van Leenwenhock, pioneristic constructor of microscopes of the XVII century). Anton, the Shaw's machine, contains 512 special chips, expressively planned, in ach of whose there are, among other things, 32 specialized antithetic pipelines to 28 states properly planned for the ultra-rapid calculation of the interaction between particles couples of the simulation of molecular dynamics. Each pipeline, in his turn contains a great number of little arithmetic units, and it is capable to produce in each cycle of the cycles of his clock to 800 megahertz a result that on a conventional microprocessor would require about fifty operations. Operating together, the pipelines incorporated in the 512 Anton's chips can effect so the most onerous portion in terms of calculation of a simulation of molecular dynamics to an effective speed of peak of further 650.000 milliards of operations to the second".

11. Discourse on the method, Reunited Editors, 1996, p. 50. F. Alquié took care of the Oeuvresphilosophiques in three volumes (Paris, 1963, 1967, 1973), grouping around the written, ordinated chronologically, the letters of the same years. A large choose is that done from A. Bridoux for the Bibliothèque de la Pléiade (Descartes, Oeuvres et lettres, Paris, 1966). It is to make a signal, moreover, that the re-edition of the Oeuvres (Adam-Tannery) excited in the 1967, a project of manual indexation, after transforming, with the concourse of the Centre de Lexicologie Politique (E.N.S. de Saint-Cloud), in a program of automatic textual analysis of the Cartesian work, conduced from the "Equipe Descartes" under the direction of J. - R. Armogathe. In the ambit of this program of research has been yet elaborated from P. A. Chane the Index du Discours de la méthode de René Descartes, Rome, 1977. The first waste harvesting of Cartesian text in Italian language has been published in the Opere, at care of various translators, $2 \mathrm{vv}$. Bari, 1967 and ri-edited, with the title Opere filosofiche, in the 1986, in 4 volumes so articulated: 1. Frammenti giovanili; Regole per la guida dell'intelligenza. La ricerca della verità mediante il lume naturale. Il mondo o trattato della luce. L'uomo, Discorso sul metodo (this last in the new translation of M. Garin, to that it is given also the version of Il Mondo e L'Uomo, works not included in the edition of the 1967); II. Meditazioni metafisiche. Obbiezioni e risposte; III. I principi della filosofia; IV. Le passioni dell'anima, Lettere sulla morale. Colloquio con Burman (under the title of Letteresulla morale has been picked the letters with Elisabeth of the Palatinate, Christine of Sweden, and with the ambassador Chnut, of the years 16431649). The long bibliographic note of E. Garin, that introduced the edition of the 1967 , has been eliminated, substituted from very brief notes to the texts, and edited at part with the title Life and works of Descartes, Bari, 1984 (1986).

12. K. Popper, Logics of the scientific discovery, p. 113.

13. At p. 150-151 exists a fair and sensible distinction between probability and statistic for the relative frequencies, in consequence of the fact that it is an asynthotic deduction of the formal logics of Hilbert.

14. Logics of the scientific discovery, p. 163.

15. Logics of the scientific discovery, p. 257-275.

16. Logics of the scientific discovery, p. 405.

17. K. Popper, Logics of the scientific discovery, p. 358. For the elements of construction of the type analysis, see P. Odifreddi - Pythagoras, Euclid and the born of the scientific thought, The library of Repubblica, 2012, p. 35-40.

18. The British journal for the philosophy of science, volume 59, number 3, september 2008, article of F. A. Muller e Simon Saunders, Discerning fermions, composite physical systems of similar particles, p. 509.

19. The British journal for the philosophy od science, volume 59, numero 3, settembre 2008, articolo di V. Allori, S. Goldstein, R. Tumulka and N. Zanghi, On the common structure of Bohmian Mechanics and the Ghirardi-Rimini-Weber Theory, primitive ontology and symmetry, pag.365 We refer as possible as the wave function is operated in dynamics, that they exist, apart to the systems of symmetry theories, dynamics models corresponding, into a deterministic way, to a calculation of the most important mathematical parts in the logic of counters. 
- $\quad$ Since the trajectories of the PO changed according to the symmetry are still solutions. BM is symmetric under Galileian transformation, even though the corresponding wave function has to undergo more than a simple charge of variables in order to make it possible.

- When changed according to the symmetry, will again be possible probability distribution for the theory. The probability distribution on the histories, when changed according to the symmetry, is the distribution of the changed histories. In other words, the action of a transformation on every history determines the transformation of a probability distribution on the space of histories. As in the deterministic case, the wave function is allowed to change in any way compatible with its relationship to the PO. For example, consider the Galileian invariance of GRWf: let the wave function and the standardized wave function be two initial wave functions related as in the partial construction of a wave function (15), that is, by the usual formula for Galileian transformations in quantum mechanics. According to the analysis of Wigner ([1939]) and Bargmann ([1954]), these transformations on wave functions and operators are given by unitary or antiunitary operators $U$, i.e. The measurement unit and the same operators in the wave functions in the symmetry group, where $U$ is an element of a unitary-projective representation of the symmetry group [operated for every $t]$.

20. these different primitive ontologies can evolve according to either deterministic or stochastic laws. Corresponding to these possibilities we have a variety of physical theory. Bohmian mechanics (BM), Bohmian quantum field theory (BQFT), a mass density field theory with Schrodinger evolving wave function (Sm), stochastic mechanics (SM), Bell-type quantum field theory (BTQFT), Bell's version of many-worlds (BMW), a particle GRW theory (GRWp), GRW theory with mass density (GRWm), GRW theory with flashes (GRWf), and two theory with flashes governed by Schrodinger (or Dirac) wave functions (Sf and $\left.\mathrm{Sf}^{\prime}\right)$.[pag. 376].

\section{M. Potenza, The universe of Einstein, p. 79}

22. A. Einstein, Relativity, Divulgative exposition, p. 81

23. A. Einstein, Relativity, Divulgative exposition, p. 80
24. L. Boltzmann, Mathematical models, physics and philosophy, On the development of the methods of the theoretical physics, p. 110. "Properly so in the experiments done before the electric states have been always changed in others in way relatively too much slow in comparison with the enormous speed of propagation of the electricity. Hertz, after tiring preliminary experiments, which conductor thread describes himself in a way very impartial, founded some experimental conditions in that the electric states modify periodically in way so original to generate observable waves. [..] Then, when Marconi produced in a place some hertzian waves very short and, with a suitable modification of the instrument, that we have called eye for the hertzian waves, he transformed them in Morse symbols in another place far many kilometers, he did not built anything that a normal optical telegraph".

25. Russell, Introduction to the mathematical philosophy, p. 113-117.

26. Russell, Introduction to the mathematical philosophy, p. 172-176. The extensive functions of a function of $\mathrm{x}$ do not provide in fact others equivalences. See also L. Boltzmann, Mathematical models, physics and philosophy, p. 154-156.

27. G. Reale, D. Antiseri, The West thought from the origins to today 3, 1994, Publishers La Scuola, p. 537 for what concerning the comparative words of probable equations for the linguistic and conceptual establishment for the analytical philosophy, and p. 296 for the linguistics, von Humbolt, Bopp, the "Grimm's law" and the "Neogrammatics".

28. G. Reale, D. Antiseri, The West thought from the origins to today 3, 1994, Publishers La Scuola, p. 341

29. G. Reale, D. Antiseri, The West thought from the origins to today 3, 1994, Publishers La Scuola, p. 518

30. Wikipedia, voice probabilism

"The universe is quanto-mechanic: this means that, even though we could know his initial state and the fundamental laws of the matter, we could calculate only a series of probabilities for his possible stories" (M. Gell-Mann, The Quark and the Jaguar, Turin, Bollati Boringhieri, 1996, p. 44)

"However the statistical considerations of the quantum mechanics apply only at a macroscopic level. Here one 
of the interesting points of the study on the points of branching off that I have just mentioned. These demonstrates that even to the macroscopic level our prediction of the future blends together determinism and probability. In the point of the branching off the prediction has a probabilistic character, whilst between branching off points we can speak about deterministic laws". (Prigogine, The hazard's laws). "The notion of probability, introduced empirically from Boltzmann, was a bravery's act extremely productive. To more of a century of distance we begin to understand in what manner it would emerge through the instability: this destroys the individual level and that statistic, and for consequence the probabilities come to assume an inherent meaning; irreducible to an interpretation in terms of ignorance or approximation"."It is for this simple fact that there are more configurations of atoms in disorder of how many would be of organized in an interesting way. A collection of atoms, each one moves in a casual way, will assume a disordered state with much more probability of an organized configuration. [..] It is very improbable that the system could have from his turn to return to a more ordinated configuration. The absence of the growth's law of entropy is all there" (Lee Smolin, The Life of the Cosmos, Oxford's University Press, 1997).

31. Statistics mechanics and electromagnetism establish the points of arrival most matures of two different conceptions of the physical world, that we have seen few times to come to blows as the theory of continuum and theory of discret: Democrite against Anassagora, Newton against Descartes, Ampere against Faraday. The physics of the latest decades of the century is dominated by the contrast between these two conceptions, that it is possible to pick still in part to our days in the difficulty to conciliate the general relativity and the quantum physics. The opposition between the two models appeared in first place as a contrast between two physics realities: from one side the Maxwell's elaboration description from the other the ponderable matter (molecules and atoms). But the relation between ether and ponderable matter have been resulting obscure. A significant classification attempt was operated by H. A. Lorenz (1853-1928). In his model the reality is considered from three different kinds of entities: the ponderable matter, provided by the traditional mechanical properties, the ether, smaterialized support of electromagnetic phenomenons void of any mechanical property, and the electrons, that is the elementary charge quantum, that are the mediators of every interaction between ether and ponderable matter. [..] Meanwhile the theoretic physics has been debating around these arguments, to the experimental level they have been standing out manifold significant discovers in the ambit of the study of the radiations: the ray $\alpha$ (ionized atoms of helium), the ray $\beta$ (electrons), the ray $\mathrm{x}$ (electromagnetic waves). This provided some materials of the theorical reflection and to the elaboration of the first atomic models, the first of whom was proposed towards the 1904 by J. J. Thomson (1856-1940), that supposed the existence of a compact sphere positively charged, in that have been inserting the electrons, hold by an electromagnetic force. But E. Rutherford (1871-1937), observing diffusion phenomenons of ray $\alpha$ with whom he bombarded a thin metallic sheet, concluded that the matter is not uniformly full and proposed in the 1911 his model, in which the atom is conceived as a solar system in miniature, at the internal it is collocated a nucleus in that it is concentrated the great part of the atom's mass; and his positive charge, and around it they wheel the electrons in circular orbits, bound from the electromagnetic force. Therefore the planetary model was bound towards an heavy difficulty, because it contrasted with the electromagnetism's principles. In fact the electron, in motion inside the electric field produced by the nucleus, it had to irradiate energy under shape of electromagnetic waves and, losing energy, it had to throw down as a spiral in the nucleus. The situation of embarrassment will be solved in 1913 by some substantial modifications [..] from Bohr and the Rutherford's atom".

32. The Michelson and Morley aim was that to measure the time's interval employed from a light ray, to achieve a going and return travel between two points separated by a rigid distance, that is to say independent either from the place or from the motion achieved from the two points in the middle in quietness. In other words, it was necessary to calculate the numeric volume of $\mathrm{v}^{2} / \mathrm{c}^{2}$, that, as we know, Maxwell considered impossible to determine, having make use of an optical instrument said interferometer; this measured the time employed by a light ray given out on the stroke ion (called emission's flat) to achieve a stroke of space from the emission point a until a mirror put on the vertical that touches in the point $b$ and then the return travel until the measurement instrument, putting in account that the reference's system is collocated on the earth and then it moves; the point of arrival a' of the ray will coincide not with the emission point a. the light ray therefore spread even in a parallel direction to the emission flat sa and even here it achieves a stroke 
until the point $\mathrm{c}$ before to be reflected from another mirror and to turn back. The task is to measure the difference between aba' and aca': the experiment gave a negative result, in the sense that the effective difference was widely lower to that foreseen mathematically, according to Michelson and Morley lower to the twentieth part and maybe more (until the fourteenth part). Around this difficulty they worried the physicians to go out from the embarrassment's situation, contriving different hypothesis: that however resulted fair, although for any disconcerting, was that it was necessary to forsake the trust in the rigidity of the length. A solution seemed been reached at the internal of the electron's theory of Lorenz, who moreover criticized hardly the experiment of the interferometer attributing the outing of phase between the provided data and that verified to measurement errors. He, considered the electromagnetic structure of the matter, assumed that its mechanical qualities could have to be altered from the motion through the ether. The translator motion will have been producing a deformation in the orbits of electrons, that it will have been translating to microscopic level in a contraction of the lengths in the direction of motion and in an altering in the measurement of the speed, the mass and the time, so that the experiment of the interferometer have been justified keeping the postulate of invariance, as regards the study of the interaction between molecules and atoms, that have taken in account the interaction among charged particles. The negative result of the Michelson's experiments was justified from the fact that the different speeds of light in the different directions were compensated by the contraction, moreover not been in relief since shared also by the straight edge that we have been employing to verify it.

But the re-writing, from the Lorenz's side, of the field's equations of Maxwell (according to a shape very much effective and compact done to Hertz and others) has been resulting disconcerting because, after to be established for a fixed system of coordinates, translated for a mobile system that moves on behalf of the first with speed $v$ on behalf of the axis of $x$, they have been requiring the introduction of a new parameter that Lorenz baptized local time. This last does not depends only by the time, but from a series of others elements whose the light speed, that of the system of reference in motion, the place, but his nature was still all to decide.

33. K. Popper, Logics of the scientific discovery, p.331-391
34. K. Popper, Logics of the scientific discovery, p. 335

35. K. Popper, Logics of the scientific discovery, p.362

36. K. Popper, Logics of the scientific discovery, p. 390-405

37. K. Popper, Logics of the scientific discovery, p. 289

38. Mathematical analysis 2, p. 399-401

K. Popper, Logics of the scientific discovery, p. 290

N. Abbagnano, G, Fornero, Philosophy's itineraries, volume 3A, Paravia, 2003. "The classification, on the comptiane encyclopedia of sciences obeys to an historical and logic criterion at the same time. In fact, it ordinates the sciences a) according to the historical sequence in that they have been achieved the positive status (historical criterion) and b) according to their passing from the simplicity to the complexity, in other words according to the rule of the increasing complexity and the decreasing simplicity (logic criterion). Such encyclopedic results been constituted from five fundamental sciences: astronomy, physics, chemistry, biology and sociology. ND. Of this hierarchy it does not take part, as it is possible to see, neither the mathematics, or the logics, or the psychology. The reasons of the exclusion are different. The mathematics has been excluded not because it will be not a science (on the contrary, it has been the first to entry in the positive state) but for the reason it stay at the basis of all the others sciences. Such a worthy is true that in the comptiane encyclopedia can be globally articulated according to the following order: 1) mathematics, 2) astronomy, 3) physics, 4) chemistry, 5) biology, 6) sociology. The logic has been excluded because Comte establish that it will not subsisting in general and in abstract, but it had identified with the concrete method employed by every specific branch of the knowledge. The psychology has been excluded seeing that Comte, taking in discussion its scientificality, sustains that the person can reach not, above himself, an objective point of view (that is we have to divide himself in two). That there is of scientific matter in the psychology, from one side it is brought again to the psychological exam of the brain (that is to the biology) and from the other to the exam of his social behavior (that is sociology). 
1. Metaphysics for absurd, pages 162-190. The type of set in the demonstration theorems includes some belonging relationships, if the set that has $\mathrm{A}$ as inieunt and B as exeunt are more great of the power of A, they exist some methods to compare the cardinality of sets in $\mathrm{C}$ language more than numerable (according to the modern language).

2. Metaphysics for absurd, p. 162-190. The type of set in the demonstration theorems includes some belonging relationships, if the set that has $\mathrm{A}$ as inieunt and B as exeunt are more great of the power of A, they exist some methods to compare the cardinality of sets in $\mathrm{C}$ language more than numerable (according to the modern language).

39. Mathematical curiosities of the professor Stewart, I. Stewart, The Sciences, 2010, p. 120

40. P. 111 and p. 227-237

- M. V. Rovighi, Elements of philosophy, Third volume, La Scuola Publishers, Brescia, 1963, p. 30. Naturally this is not at all the Russell's opinion who, following Frege, try instead a nominalistic definition of the number. About Frege we had now translated in Italian, at care of Geymonat, Die Grundlagen der Arithmetik, together with some other writings in the volume that takes the title Arithmetics and Logics, Turin, Einaudi, 1948.

- The relational plan is an interpretative model or else a relational grammar of the voices to be classified as given in levels or states of the interpretative scheme. In fact, the geometry and the objectualisation of the reality are some instruments of internal analysis of the grammar, given that, to the levels of the computers, they exist morphosintattic analyzers that places beyond the geometry and the objective data. It exist after a mathematical linguistics, that by means of that quantitative, is able to accomplish an ordinate subdivision of the grammar in analysis through the structural regularity, it is based on the information's theory and on the interpretation of qualitative data.

- A. Einstein, The revolution of the contemporary physics, T. Damour, p. 76-118 "The terms of fifth order unwind a particular role: as the calculations show, they are caused from the part of the gravitational interaction that propagates to the light speed between the two objects, in other words they reflect the waves existence". Studying the effect of these terms on the motion of a binary pulsar, it is possible to find that they cause a progressive acceleration of the orbital frequency of the system, that is a progressive decrease of the orbital period".

41. Piero Angela, Travel in the science, from the Big Bang to the biotechnologies, Mondadori, 2002, p. 64

42. Piero Angela, Travel in the science, from the Big Bang to the biotechnologies, Mondadori, 2002, p. 285. "The De Broglie's theory, that considered the atomic particles to the rate of waves, and the Heisenberg's determination principle, constituted the foundation of the quantum mechanics, one of the most important scientific revolution of the $\mathrm{XX}$ century. Until this moment, the scientists was convinced that the physical systems would be deterministic: measuring with precision the initial conditions and knowing the exact equations, it would be possible to foresee with precision their future evolution. With the birth of QM, the scientists must to renounce, at least in the atomic microcosme, to the wish to foresee with absolute precision and uncertainty every phenomenon. It is necessary to be satisfied of the probability. This means not, therefore, that in the world of the atom have been reigning the confusion and the uncertainty. The quantum mechanics has revealed able to explain all the atomic phenomenon and represents one of the greatest successes of the modern science".

43. D. Leavitt, Alan Turing and the discovery of the computer, The Sciences, 2009, p. 35

44. Informatics, p. 324

45. Sciences, Garzanti, voice physics. The optics, developed itself in independent way as study of the bright radiation, is brought again to the concept of propagation of the electromagnetic waves (in fact the light presents characteristics of electromagnetic wave) and re-enter then in the electrodynamics. When the geometrical and physical characteristics of the mean in that the light propagates are such it is possible to disregard the wave's length, the study of phenomenon happen, with good approximation, in the ambit of the geometrical optics; this is equal leaving out of consideration the undulatory effects and considering the light as a ray that propagates in straight line. When 
therefore the conditions for the acceptance of the geometrical approximation are not satisfied (and they verify phenomenon of diffraction, interference, etc.) it is necessary to recur to the undulatory optics. See also voice electrochemistry.

46. The problems of the millennium, p. 159

46. 1. P.178-188, through the problem $\mathrm{P}$ versus NP, and the Poincaré's conjecture, that are parts of the same logics, we must to compose a mathematical problem in function, that it would be algebraic or mathematics, to understand what is his system of reference, in the computer, for example, what is the first and the second term to calculate the series of the numbers. At this point, the computer provides to us the mathematic part, but that algebraic must to take part of a statistic method. The model of choose provides two types of systems: the system of the number in $\mathrm{R}$, and the system of descriptive information, that in the computational theory is unrolled though different models of logics.

47. Mathematics, basic course, p. 120-124, p. 135 and p. 247.

48. if to a distance of time the same entrance are provided the system gives back the same gettings out

49. The problems of the millennium, p. 203-212. "In consequence, in the course of his career, Poincaré had worked still over functions including complex numbers, and generally speaking on recognize to him the worth to have given origin to the theory, immensely important, of the analytic functions of differential complex variables. Several times, he applied own talents even to the study of the numbers theory and geometry. Here, therefore, to interest us indeed is his work in that branch of mathematics called "topology". It is in this field that stands out in fact the fifth millenium problem: the Poincaré's conjecture. Although the topology had been sinking its roots in the Gauss work and others yet at the middle of the nineteenth century, his really beginning is collocated only in 1895, when Poincaré introduced practically all the concepts and the fundamental methods that have been given impulse to the new discipline in the following fifty years. The topology is a sort of "ultrageometry", issued from the usual geometry and infinitesimal calculation, in which the mathematician studies properties very general of superficies and other mathematical objects".
50. The problems of the millennium, p. 154-157.

51. G. Zwirner, L. Scaglianti, Infinitesimal analysis 3, Functions in R, Cedam, 2004, p. 382.

52. P. 383

53. I. Newton, the gravity, the light and the colors of the world, The library of Repubblica, p. 41-42.

54. F. Tottola, A. Allegrezza, M. Righetti, New course of chemistry, Minerva Italica, 2005, p. 86. See also p. 189.

55. The British Journal for the Philosophy of science, volume 60, number 4, December 2009, article of R. Healey, Perfect Symmetries, p. 705.

55. 1. The British Journal for the Philosophy of science, volume 60, number 4, December 2009, article of T. Button, SAD Computers and Two versions of the Church-Turing Thesis.

56. Informatics, p. 144-150.

57. Informatics, p. 239 and p. 78-85 for the second order, and p. 175-179 for the distribution's method.

58. It is possible to see the main rules of the method at the p. 72 of the Explanation on the method of Descartes.

59. Complex and organized, (p. 41). Definition of "all", "part" and "summarization". In the natural sciences the concept of addiction is in two typologies: scalar or vectorial (of one of more dimensions, until also to the tensors), and in this case the addiction is defined in way the result would be the summarization of the parts. That, as well noticed, is worth for the extensive properties. They exist other properties, called intensives, for which the summarization is not defined. An extensive property is the weight, one intensive is the temperature. To put together two objects of a kilogram, gives a whole of the weight of two kilograms, whilst put together two objects at a temperature of $20^{\circ}$ centigrade gives a whole that is still at $20^{\circ}$. We notice they exist properties, as the volume, that are extensive and, then, additive ( 3 litres +4 litres $=7$ litres), but when it happens a chemical reaction the weight keeps itself, the volume can keep itself or not. This fact could take in advise it is not obtained an aggregate of gases, but a new system with an its volume. Obviously Nagel does not speak of these chemical cases, as it is usual in all the epistemologists. 
- $\quad 80)$ Coming to the chemistry, we ask if they exist or less its specific laws. In literature, this question is considered still open. According to Caldin, they exist in chemistry more kinds of laws:

- $\quad$ Functional relationship among variable properties of a given system (for example the dependence by the temperature of the specific heat of a pure substance or the constant of speed of a reaction).

- Laws that establish the existence of materials with reproducible properties. In this category should to re-enter the laws that regulate a chemical reaction $(\mathrm{A}+\mathrm{B}$ under some conditions gives $\mathrm{C}+\mathrm{D}$ ).

- The periodical law (the properties of the elements are periodic function of their atomic number).

- The law of the gases and different rules about the chemical reactivity.

(p. 31). The Prigogine's work try to explain the relative stability of ordinated systems and, at times, in such a way ordinated in a world governed from the second law of the thermodynamics and it makes this through the concept of dissipative system [..]. According to Prigogine, for the open systems we can affirm that

1. the stationary states are not defined from the maximum thermodynamic greatness that expresses the tendency of the closed systems and in thermal words isolated to evolve towards an equilibrium in thermodynamics, but from the approach to the production of this greatness in a minimum value.

2. the greatness can diminish in these systems.

3. the stationary states with a production of greatness minimum are, generally speaking, stables. For this reason, if one of the variables of the system changes, the system shows some transformations in the opposite sense. It is understanding so why the Le Châtelier's principle would demonstrate validated, not only for closed systems, but also for the ones open systems.

4. the consideration of the irreversible phenomenon leads to the thermodynamic time's concept in antithesis with the one astronomic concept.

(p. 121). In this representation the extensive greatnesses have the role of independent variables, whilst the ones intensive greatness are introduced as derived greatness. This fact is in a direct opposition with the practical situations that present in laboratory, since the intensive greatness are more easily measurable and controllable and they assume then the role of variables operatively independent, whilst the ones extensive become, from the operative point of view, some derived greatness. This affirmation results more than ever true in the case of the temperature and in the case of a thermodynamic greatness: it does not exist, in fact, any instrument that would be able to measure the greatness, whilst thermometers and thermostats, used to measure and control the temperature, take part of the common endowment of every laboratory. It is asked, then, if it would be possible to re-handle the mathematical formation in such way to make appear the intensive greatnesses as independent variables. A re-formulation of this kind not only is possible, but allow us to introduce some new representations in thermodynamics. It goes however underlined that the thermodynamics is a branch of the autonomous physics and complete in his logical structure and that this own characteristics does not depend on the used representation and, by consequence, the introduction of the representations in a question of pure commodity. Sure is without these new representations the thermodynamics would be practically unusable from the practical point of view, but they are not completely indispensable for the logical construction of the theory (p.. 115-123).

60. Leibniz, Philosophical written of G. W. Leibniz, volume II, New essays on the human intellect and preparatory essays, Utet, at care of Bianca, 1967, p. 420-439, and p. 700-706. Here the author explains on 
the argument. "About the names of the simple ideas, of the relationships, of the name of the substances", of the definition of the number, and then of the phenomenology in different states, between mathematical philosophy and physics. Analogously to the d'Holbach baron [Utet, 1978, p. 131] on take reference to the general result of the sum of the elements, in analogous way to the Cartesian mechanics, which physicalisme comes to be expressed in the mathematician mechanicisme of Descartes. With the purpose to make at an accomplishment a demonstrated geometry, the efforts in the elaboration of d'Holbach are addressed to integrate the definition of number.

60. 1. Philosophical encyclopedia Bompiani, voice logics, p. 6556, "The idea of the algebra of the logics was been overshadowed from J. Bernoulli in the 1685 and often partially realized from Lambert, Plouquet, Euler, but his paternity is regularly attributed to Boole for the impact that his work, moreover more articulated, had on the following logicians. Boole (The mathematical analysis of Logics, Cambridge, 1847), to every class $\mathrm{x}$ associated an operator $\mathrm{x}$ that selects from an universe of objects (indicated with the symbol 1) the ones that are $\mathrm{x}$. "xy" selects the $\mathrm{y}$ from what that $\mathrm{x}$ selects. (In a second time, Boole worried to give a psychological explanation of these acts). For the selection is worth the commutative law and the fact that to repeat a selection does not add anything of new $(x x=x)$. (Subsequently, in An investigation of the Laws of Thought, edited in London in the 1854, he used the symbols $\mathrm{x}, \mathrm{y}, \mathrm{z}$...to indicate directly the same classes; mean with "class" also the "universe" and "void", indicating with 1 and 0 respectively). In difference with what happens in the numeric algebra, generally speaking it is not admitted here an analogous operation with the division, to avoid wrong conclusions (for example, if from $x y=y z$ would be concluded $x=$ $y$, from the fact that the class of the celibate postmen would be co-extended to the class of the blonde postmen, it would be concluded that the class of celibates is co-extended to the class of blondes), but it is given a kind of its substitute, the abstraction $x=y z$ can be written as $\mathrm{x} / \mathrm{y}=\mathrm{z}$, meaning $\mathrm{z}$ denotes a class that is obtained abstracting, from the belonging as a number to the class called $\mathrm{x}$, the restriction of the being included in the class called y. Example: "class of the men"/ "class of the rational being" = "class of animals". Moreover, " $x+y$ " is defined only partially, when $\mathrm{x}$ and $\mathrm{y}$ do not have any in common, and " $\mathrm{x}-\mathrm{y}$ " is defined only if $y$ is part of $x$. The propositions are represented by equations: for example, "All the $\mathrm{X}$ are
$Y$ " is represented from " $\mathrm{x}=\mathrm{xy}$ ", because the selection of the $\mathrm{X}$ from the $\mathrm{Y}$ gives $\mathrm{X}$ if and only if all the $\mathrm{X}$ are $\mathrm{Y}$, whilst "Any $\mathrm{X}$ is $\mathrm{Y}$ " is represented from " $\mathrm{xy}=0$ " (where "0" represents "void"). With the aim to introduce the affirmative universal proposition (A), affirmative particulars (E), negatives universals (O) and negative particulars (I) of the traditional formal logics as equations, Boole adopted the indefinite symbols $\mathrm{v}$ and $\mathrm{w}$, to indicate generically that they exist numbers of the class which the ones symbols are applied. A $x=v y E=x=v(1-y) I v x=w y ~ O v x=w(1-y)$ or also $A x(1-y)=0 E x y=01=x y=v O x(1-y)=v$.

The treatment of the syllogism happens trying to express the introductions in one of these shapes, after these are combined to eliminate algebraicly $y$ and on solve in accordance with the term-subject. That is to say the inferences are achieved for substitution and replacement of equal words; so, from " $\mathrm{x}=\mathrm{xy}$ " and " $\mathrm{y}=$ $y z$ ", it is possible to obtain $x=x(y z) z=x z$, that is the syllogism AAA of the first figure. Boole noticed that his system is not limited to an interpretation for classes. If the ordinary algebra is restricted to the case in that the only possible values for $\mathrm{x}$ are 1 and 0 , ever the law $\mathrm{xx}=\mathrm{x}$ will be worth and, for this reason, this will be an interpretation of the system. Another is in the words of the propensity's logics: laid down that 1 and 0 would represent respectively the true and the false, and $\mathrm{x}$ and $y$ propositions, $x y=1$ will signify the truth of their conjunction, $x+y=1$ the truth of their exclusive disjunction, $x(1-y)=0$ the fact that if $x$ is true then also $y$ is true. In Boole a theory of the relationships missed: C. S. Peirce was the one to present in various essays, in axiomatic way, an algebra of the relational logics (and not relational) combining the Boole's work and that of the Morgan, for whom he invented particular technical instruments (as the premised shape, in that all the quantifiers come before the remainder of the formula in that they appear) and he suggested the Church's theorem (there is not mechanical proceeding to determinate the validity of an argument in that relationships and quantifiers appear). Jevons (Pure logics, London, 1864) tried to eliminate from the Boole's theory all the aspects that did not have a fair meaning: the division, the letters $\mathrm{v}$ and $\mathrm{w}$, and he admitted the summarization also in the case of not disjoined classes. J. Venn (Symbolic logics, London, 1881) from a side he tried to recover the first idea of Boole in accordance with the exemplification achieved by Jevons, trying a meaning for the division (in the words intending $\mathrm{x} / \mathrm{y}$ as a partial function one-to-many of $\mathrm{x}$ and $\mathrm{y}$, that is seeing it as the one class - and there 
is more than one - such for that the intersection of $x y$ and $\mathrm{y}$ is identical to $\mathrm{x}$ : this happens only when $\mathrm{x}=\mathrm{xy}$, that is when all the $\mathrm{x}$ are $\mathrm{y}$ ), from the other he improved the diagrams - introduced yet in the Seven hundred from Euler - and he utilized them to evaluate the truth of the categorical syllogisms. For reasons of opportunity), he choose to intend the categorical propositions (all the $\mathrm{X}$ are $\mathrm{Y}$ ) as "the class of the things that are $\mathrm{X}$ and not-Y is void" so, the circles used from him to represent the classes and their relationships of intersection and inclusion were traced however (in difference with what did Euler, since he did not traced the void class and he used to shade the void regions and to make a bar on that they did not have exact existential information).

In Germany, the first half of Eight hundred saw to reign the Hegelian statement, that identifies logics with metaphysics: the logics has for object the thought and this identifies with the reality. Meanwhile, nevertheless, it appears the publication of the lessons of the Bohemian Bernand Bolzano (ecc.). He, in addition to a particular transposition of all the propositions under the shape "A has B" (for that "this is golden" becomes "this has the property to be gold". " $\mathrm{X}$ is not starving" becomes "X has the want-of-hunger ecc.) he proposes interesting innovations in the evaluating the truth of a proposition: he introduces a concept very similar to that tarskian of "relationship by consequence", expressed as "derivability on behalf of a certain class of terms", saying that Q derives from B if and only if $\mathrm{Q}$ is coherent and every model of $\mathrm{B}$ is model of $\mathrm{Q}$ on behalf of that class of terms (the difference between its notion and that tarskian consists in the clause of coherence). In Germany it comes to maturity also an attention towards the formal logics with F. A. Trendelenburg and after W. Windelband (that nevertheless criticized the modern English modifications, as the quantification of the predicate) and it is present a trend of thought (which they belonged, among the others, W. Wundt and Ch. Sigwart) that founded the normativity of the formal logics on the psychology. Meanwhile, E. Schroder, on the Boole's wave, utilizing the further contributions deriving from the theory of the relationships of Peirce, achieved analogous work of elaboration of a "formal algebra" as "preparatory to studies on the most various numeric systems and operations of calculation that should be invented for particular purposes", considering the logic calculation as model for the formal algebra (Vorlesungen uber die Algebra der Logik, Leipzig, 1890-1905). It has been then in mathematical field, more than philosophical, that it is happened the resurrection of the formal logics in Germany and in Great Britain in the Eight hundred. In the meantime, the development of the analysis (that is to say the study of the continuous, and the real numbers) it was having given back opportune and necessary the drawing up of manuals that would expose of it instructively the results and then it presented the opportunity to reflect on the "points of leaving" of the same theory. Previously, the real numbers were founded on the straight line, indicating the single points. Such proceeding did not seem more such sure at the epoch, since the not-Euclidean geometries (they did not accept the $\mathrm{V}$ postulate of Euclid, called "of the parallels", or because they were admitted for a point more parallels to a given straight line, or because they were denied the existence of the parallels), with their same presence, they were given back the geometrical reference less solid. So various attempts were achieved to bring again the real numbers to the natural numbers, though definitions in terms of sections of Dedekind or limits of converging progressions, as proposed whether by Cauchy or by Cantor, that became after famous as father of "ingenuous" theory, not formalized, of the set of samples (Beitrage zur Begrundung der transfiniten Mengenlehre, Leipzig, 1894-1895).

61. Article of P. Odifreddi, The instruments of the geometrician, The Sciences, may 2014, p. 18

62. F. Dyson, The scientist as a rebel, The library of the sciences, 2009, p. 156

63. F. Dyson, the scientist as a rebel, The library of the sciences, 2009, p. 171

64. The British Journal for the philosophy of science, volume 61, number 4, December 2010, Article of W. Aitken and J. A. Barrett, A note for the Physical Possibility of Transfinite Computation, p. 867.

The British Journal for the philosophy of science, volume 60, number 4, December 2009, article entitled Perfect Symmetries, of R. Healey, p. 705. See Appendix A, in the same article, at p. 715.

65. Article of P. Odifreddi, A travel in the geometrical results a lot known of this giant of the science, p. 22, and article of A. Alexander, The secret of the history of the calculation, The sciences, June 2014, p. 50. 
- P. Odifreddi counts Pythagoras, Euclid and the born of the scientific thought, The library of Repubblica, 2012, p. 12, regarding also a variable of the algebraic equation.

- Epistemology, An Italian journal for the philosophy of science, 2009, volume 32, number 2, p. 260-269.

- Dictionary of linguistics, and philology, metrics, rhetoric, of G. I. Beccaria, Einaudi, 2004.

- Warburton, The first book of philosophy, $\mathrm{p}$. 150-160.

- P. Odifreddi counts Einstein and the relativity, The library of Repubblica, 2011, p. 63. "The special relativity's theory conciliates with success the new experimental data with the classical mechanics of an inertial reference's system (in that the celestial bodies move of rectilinear uniform motion in other words), whilst the extension to all the reference's system is confronted from the general relativity's theory. [...] More besides they are exposed some empirical proofs in sustain of the theory, in confirmation of the fact that whatever scientific theory must could be subdue to the proofs of the experience". In the fourth part of The evolution of the physics the other great theoretic intuition of Einstein has been confronted that, together with the relativity's theory, is at the basis of the contemporary physics. On traits of the quantum's mechanics, describing the physical phenomenon in that the relativistic component can be unaware. Einstein will be engaged a long time, and vainly, to arrive to the unification of these two great theoretic buildings".

- Popper, Logics of the scientific discovery, $p$. 248-257 and p. 265-274. See also Russell, Introduction to the mathematical philosophy, p. 141-149 on the incompatibility and the theory of deduction."Kant, having observed that the geometricians of his time did not demonstrate their theorems with alone reasonings, but they make call to the figures, he invented a theory of the mathematical reasoning according to that the deduction is not ever rigorously logic, but it requires always the support of that he called the "intuition". All the development of the modern mathematics, with its increasing research of rigour, is gone in the opposite sense to the Kant's theory". Hume, Research on the human intellect, translation of Mario dal Pra, Publishers Laterza, 1996. "The geometry helps us in the application of this law, providing to us the exact dimensions of all the parts and all the figures that can entry in some kind of device; but the discover of the law is due therefore just to the experience and all the abstract reasonings of the world could not never to make an advance to us of a stride towards his knowledge". "All the reason's objects and these of the human research can be naturally divided in two kinds, that is relation of ideas and matter of fact. To the first kind belong the sciences of the geometry, the algebra and the arithmetics; and, in short, whatever affirmation that would be sure by intuition or demonstration's way to know".

Dictionary of linguistics and philology, metrics, rhetoric, directed by G. L. Beccaria, Einaudi, 1994, to the voice connective. In logics, expressions that, combined with one or more enunciates, generate a new enunciate more complex. A connective is unary, binary, ternary, etc. according to it would combine itself with one, two, three enunciates, etc. Particularly important are the connectives that denote functions of truth (connectives true-functionals); on traits of the ones connectives that generates complex enunciates which truth value depends just from the truth value of the constituting enunciates. The connectives truefunctional of more common use are 1) the negation, that can be indicated with the symbol; given a whatever enunciate $\alpha$, ("not a") is the enunciate that results true if $\alpha$ is false and vice versa; 2) the conjunction, that can be indicated with \&: given two whatever enunciates $\alpha$ and $\beta, \alpha \& \beta$ (" $\alpha$ and $\beta$ ") is the enunciate that results true if $\alpha$ and $\beta$ are both true and false otherwise; 3 ) the disjunction, indicated with $\mathrm{v}$ : given two enunciates $\alpha$ and $\beta, \alpha \vee \beta$ (" $\alpha$ otherwise $\beta$ ") is the enunciate that results false if $\alpha$ and $\beta$ are both false and true otherwise; 4) the involving, indicated with : given two enunciates $\alpha$ and $\beta, \alpha \beta$ ("if $\alpha$ then $\beta$ ") is the enunciate that results false if $\alpha$ is true and $\beta$ false, and true otherwise. Between the connectives not true-functional studied by the logicians they can be quoted the modal operators, that correspond to expressions of the natural language of the type of "it is 
possible that", "it is necessary that", etc. In linguistics, it is equal to the conjunction.

70. The British journal for the philosophy of science, volume 60, number 4, December 2009, Article of T. Button, SAD computers and Two Versions of the Church-Turing Thesis, p. 765.

71. K. Popper, Logics of the scientific discovery, p.209-224, with regard to a probabilistic system of speculative metaphysics. "Other cases to it can be applied the probability's theory, such the statistic floatings or the statistics of individual events with casual character, are reducible to the case that we have discussed; to the case, that is to say, of the microeffects measurable with precision. For statistic floatings I mean phenomenon as the Brownian motion. Here the interval of precision of the measurement $(+\varphi,-\varphi)$ is more small of the interval $\Delta \mathrm{p}$ characteristic of the number $\mathrm{n}$ of the micro-events that contributes to create the effect; then we must to wait, as highly probable, deviations measurable from $\mathrm{p}$. The fact they would have place such deviations will be controllable, because the same floating become a reproducible effect; and to this reproducible effect are applied my reasonings of before; according to my methodological requests, besides a certain greatness (besides a certain interval $\Delta \mathrm{p}$ ) the floatings must to be not reproducible; neither they must be it the long sequences of floatings in an only and same direction, etc. corresponding arguments are worth for the statistics of the casual events (p. 217). Somewhere else "however, in more technical sense our definition is not circular. His definiens works with an intuitive idea perfectly fair: the idea of the varying the initial conditions of our world; for example, the distances among the planets, their masses, and the mass of the Sun. He interpreters the results of these changing: as the building of a kind of "model" of our world (model or "copy" that must not to be necessarily faithful in accordance with the initial conditions), and then it imitates the well noticed artifice consisting in calling "necessaries" the ones assertions that are true in the universe of all these models (that is for all the initial conditions logically possible). [..] In spite of this, in difference with Kneale, I consider "necessary" a pure and simple word, an useful label to distinguish the universality of the laws from the "accidental" universality. Naturally, whatever other label would give back exactly the same service, because here of relationships with the logic necessity there are not many. They are mostly in accordance with the spirit of the wittgeinsteinian paraphrase of Hume
"A pressure, according to that a think should to happen because it happened another of it, there is not. There is only the logic necessity". a b with is connected with the logic connection only in a way: the necessary link between $a$ and $b$ can be not found neither in $a$ or in $b$, but in the fact that the conditional ordinary corresponding (or "retrieval implication a b without "N") follows with logical necessity from a nature's law; that is to say it is necessary relatively to a nature's law". (p. 492-497).

72. Russell, Introduction to the mathematical philosophy, p. 45-53.

73. K. Popper, Logics of the scientific discovery, p. 480-481.

74. Russell, Introduction to the mathematical philosophy, p. 187-190 regarding probabilistic assertions on the grounds of the rules called functions of the system in mathematical analysis.

75. K. Popper, The purpose of the science, Armando Editore, 2000, p. 81, regarding the realism in logics. Russell, Introduction to the mathematical philosophy, p. 115. 